Richard Schrodt (Wien)

\title{
Von den Kräften der deutschen Sprachkritik
}

\begin{abstract}
Johann Leo Weisgerbers bekannter Titel bezieht sich auf Humboldts EnergeiaBegriff, also auf die Sprache als wirkende Kraft. Auch in diesem Beitrag soll den wirkenden Kräften nachgegangen werden, freilich nicht als Unterstellung eines wesenhaften Sprachvermögens, sondern als Versuch, die wirksamen Motive der sprachkritischen Einstellungen, Publikationen und publizistischen Erscheinungen an einem Raster sozialwissenschaftlicher Begriffe darzulegen. An einigen ausgewählten Presseberichten und grammatischen Beispielen (Veränderungen im Bereich der deutschen Zeitenfolge) wird zunächst gezeigt, dass sich Sprachkritik oft schon von ihrem Gegenstand, der deutschen Sprache, weitgehend gelöst hat. Auch angesichts neuer Formen von substandardsprachlichen Erscheinungen (z.B. Jugendsprache, Jargon, Kiezsprache usw.) kann oft nachgewiesen werden, dass es sich in vielen Fällen um kommunikativ funktionale Sprachformen handelt. Um es schlagwortartig zusammenzufassen: Es gibt Sprachkritik ohne Sprache. Die „wirkenden Kräfte“ der Sprachkritik sichern vielmehr die Wahrnehmung gesellschaftlicher Differenzen und machen damit das Gefüge unterschiedlicher Lebensformen deutlich. Sie werden hier mit systemtheoretischen Begrifflichkeiten nach Niklas Luhmanns Theorie sozialer Systeme beschrieben und damit auch erklärt. Während das für die 80erJahre des vorigen Jahrhunderts charakteristische Programm der „Kritik der Sprachkritik" auf eine sprachwissenschaftliche Aufklärung zielt, scheint heute vielmehr eine soziologische Aufklärung diese metakritische Funktion erfüllen zu können. Es könnte sich aber auch zeigen, dass Sprachkritik ihren Beitrag zur Stabilisierung des gesellschaftlichen Zusammenwirkens leistet - wenn man sie nicht als Sprachkritik im engeren Sinn versteht.
\end{abstract}

\section{Strukturalismus als erklärende Methode}

1.1 „Von den Kräften der deutschen Sprachkritik“ hat intertextuelle Bezüge. Der Titel erinnert an die Arbeiten Johann Leo Weisgerbers, des Begründers der inhaltbezogenen Grammatik. Weisgerber stellte in einem Bändchen aus dem Jahr 1954 mit dem Titel „Von den Kräften der deutschen Sprache“ einige programmatische kleine Schriften zusammen, darunter die Aufsätze „Die Sprache unter den Kräften des menschlichen Daseins“ und „Die geschichtliche Kraft der deutschen Sprache“. Weisgerber verstand im Anschluss an Wilhelm von Humboldt die Sprache als „wirkende Kraft", die durch das "Gesetz der Sprachgemeinschaft" das menschliche Leben bestimmt: „Unter allen Gebilden des Gemeinschaftslebens ist die Sprachgemeinschaft die einzige, die mit fast naturgesetzlicher Sicherheit wirkt.“ (Weisgerber 1954, S. 11). Weisgerbers Konzeption einer sprachbestimmten 
und sprechbestimmenden „Zwischenwelt", die durch ihre „innere Form“ die Kommunikation in einer Sprachgemeinschaft begründet und sichert, hat seinerzeit zu bedeutenden Neuerungen und Erkenntnissen auf dem Gebiet der deutschen Grammatik geführt. Doch Ende der 50er-Jahre geriet diese Konzeption zunehmend in Kritik, bis hin zum abwertenden Schlagwort der „Weisgerber-Linguistik“ in den 60ern. Tatsächlich verführt der deutlich vorhandene geistesgeschichtliche Ansatz zusammen mit einem unbegründeten Sprachidealismus zu pseudowissenschaftlichen Hypostasierungen von Kräften, Welten, Formen und Bildern, die einer empirisch begründeten Wissenschaft entgegenstehen, und die ideologische Nähe zur „volkhaften“ und „völkischen“ Sprachbetrachtung gibt der ganzen Richtung einen modrigen Beigeschmack. Helbig hat das prägnant zusammengefasst:

Was bei Humboldt immerhin noch als kühner Ansatz zu werten ist, das komplizierte Verhältnis zwischen objektiver Wirklichkeit, gesellschaftlichem Denken und Sprache zu beleuchten, ist bei Weisgerber ein reaktionärer Rückgriff auf das Gedankengut einer längst überwundenen Epoche des wissenschaftlichen Denkens, auf die Romantik und mit ihr verbundene Kraft-Terminologie. (Helbig 1970, S. 145)

Man darf aber auch angesichts dieser Kritik nicht vergessen, dass viele Arbeiten entstanden, deren philologische Genauigkeit zu wichtigen Einsichten geführt haben - Einsichten, die heute unter dem Etikett von Sprachpragmatik, Handlungstheorie und kognitiver Linguistik durchaus als berechtigt erscheinen. Dass sich die seinerzeit in der DDR erschienen „Grundfragen der deutschen Grammatik“"von Wilhelm Schmidt (zuletzt 5. Auflage 1973) an mehreren Stellen wie eine Zusammenfassung von Brinkmanns „Die deutsche Sprache“ (1962) lesen lassen, sei als „Treppenwitz der Grammatikgeschichte" wenigstens erwähnt.

1.2 Doch hier soll es nicht um die Geschichte der inhaltbezogenen Grammatik gehen, sondern um die „Kräfte“, die hinter den sprachkritischen Aktionen stehen. Vergleichbar ist freilich der strukturalistische Ansatz. Die inhaltbezogenen Grammatiker haben immer wieder behauptet, auf der methodischen Grundlage des sprachwissenschaftlichen Strukturalismus nach Saussure zu stehen, und das zweifellos mit Recht. Das ist besonders an den Arbeiten zum Wortfeld ersichtlich, aber auch die Untersuchungen zu grammatischen Feldern und Funktionsbereichen beruhen auf strukturalistischer Methodik. „Structuralism, I am suggesting, is a response to the need [...] for a ,coherent system ' that would unite the modern sciences and make the world habitable for man again“ (Scholes 1974, S. 2). Tatsächlich: Der Strukturalismus kann als ein ganzheitliches Verfahren verstanden werden, mit dem Sprache, Literatur und Kultur auf Gemeinsamkeiten untersucht werden. Jede strukturalistische Beschreibung ist zugleich auch eine Erklärung. Mit strukturalistischen Erklärungen können auch die gesellschaftlichen Kon- 
stellationen dargestellt werden, die zu sprachkritischen Diskussionen führen - gesellschaftliche Konstellationen, die den Bereich des rein Sprachlichen weit überschreiten und somit Sprachkritik auch in ihrer Paradoxie erst verständlich machen können.

1.3 Der sprachwissenschaftliche Strukturalismus macht es möglich, sprachliche Formen und Funktionen durch ihre Zeichengestalt und mit universalgrammatischen Überlegungen synchronisch und diachronisch zu erklären - oder zumindest Erklärungsversuche so zu formulieren, dass sie im Prinzip falsifiziert werden können und damit zum Fortschritt der Wissenschaft beitragen. Strukturalismus ist aber auch eine Methode, gesellschaftliche $\mathrm{Zu}$ sammenhänge im Reden über Sprache - sei es wissenschaftlich oder laienlinguistisch - wenn auch vielleicht nicht im Einzelfall deduktiv-nomologisch zu erklären, sondern doch durch den Bezug auf soziale Funktionsgefüge und allgemeine Gesetzlichkeiten verständlich zu machen. In sprachwissenschaftlicher Sicht erscheint Sprachkritik paradox: Sprachliche Veränderungen sind in dieser Sicht notwendige Anpassungen der sprachlichen Formen an ihre kommunikativ relevanten Funktionen. Wenn es da etwas zu kritisieren gibt, dann gehört es eher zum Bereich der Stilistik, der Textsortenlinguistik und der Textpragmatik, und das sind Gebiete, in denen ebenfalls kontroverse Diskussionen vorkommen. Aus dieser Sicht kann man von „Sprachmythen“ sprechen (vgl. dazu Klein 1986). Mythen sind Bestandteile unseres kulturellen Wissens und insofern Wissensbestände, die für kulturelle Orientierungen wichtig sind, unabhängig von ihrer wissenschaftlichen Begründung. Maitz (2012, S. 4f.) hat ihre Funktionsweise klar zusammengefasst:

Sie verkörpern tradierte, für die jeweilige Kultur konstitutive Überzeugungen, die von den Trägern dieser Kultur allgemein anerkannt und akzeptiert werden. Zu ihren Eigenschaften gehört es, dass sie nicht auf rationaler Erkenntnis basieren, sondern vielmehr tradierte Glaubensinhalte darstellen; sie sind ausgesprochen dogmatischer Natur und lassen sich am ehesten als verbindliche, normative Glaubensaussagen über Sprache charakterisieren, die für die meisten Menschen deshalb auch keiner rationalen Begründung bedürfen.

In dieser Formulierung ist noch ein ethnologisches Moment der Funktion von ethnischen Begründungsgeschichten vorhanden. Für unsere moderne Gesellschaft ist es sinnvoll, sich nicht auf „die Kultur“ als Ganzes, sondern auf Teile der Kultur (welcher Art auch immer) zu beziehen und nicht allgemein von „Trägern dieser Kultur“, sondern bestimmten Schichten von Kulturträgern zu sprechen. Das ändert nichts daran, dass Sprachmythen existieren und dass sie eine bestimmte Funktion haben, die es zu erklären gilt. Das soll in den folgenden Ausführungen ansatzweise versucht werden. Ich beschränke mich auf drei Beispiele. 


\section{Sprachkritik ohne Sprache 1: Der Mythos von der Fetzenliteratur}

2.1 Zu den paradoxen Erscheinungen der neueren Zeit gehört es, dass Sprachkritik weitgehend ohne Bezug auf konkrete sprachliche Formen erscheint. Während selbst Sprachkritiker wie Karl Kraus und Dolf Sternberger, denen es vornehmlich um Gesellschaftskritik ging, immer wieder an konkreten Wörtern, Wendungen und Ausdrucksweisen anknüpften, steht dieser konkrete Bezug heute nicht mehr im Vordergrund.

2.2 Im Heft 11/2012 der Zeitschrift „GEO“ finden sich auf dem Umschlag mit dem Titel „Der Untergang der deutschen Sprache?“ folgende Phrasen, präsentiert in Schreibmaschinenschrift auf Papierfetzen: Schick mir ne simse auf mein phone / Isch ge ma nach Bahnhof, Alda / Sport fällt aus, weil die Halle ist zu / Das innovative Tool macht die Customer Journey transparent / 4. Abfahrt recht's / Stylische Boxer short, haut freundlich / Wegen Vortbildung geschlossen. Das entspricht einem Bündel von altbekannten Kritikbereichen: Neue Medien, Kiezsprache, weil mit Hauptsatzverbstellung, Anglizismen und Trendsprache, falscher Apostroph, falsche Getrenntschreibung von Wortzusammensetzungen, Rechtschreibfehler. Diese Beispiele kommen dann bis auf den weil-Satz im Artikel von Johanna Romberg nicht vor. Dort geht es vornehmlich um Informationen von Jürgen Erich Schmidt, dem Leiter des „Forschungszentrums Deutscher Sprachatlas" in Marburg. Gegenstand ist das Marburger Projekt REDE (regionalsprache.de), in dem die Dialektlandschaften und der Sprachwandel der neueren Zeit erforscht werden. Der Artikel selbst ist sachlich und für Laien durchaus informativ. In sechs Kästen werden die Themen Anglizismen, Wortgeschichte, Sprache der neuen Medien, Kiezsprache, Schweizerdeutsch und Sprachwandel behandelt, und zwar durchaus ohne sprachpessimistische Einstellung. Der in der Überschrift des letzten Kastens eingefügte Satz „Das Deutsche verändert sich, aber es wird deshalb nicht schlechter", fasst im Großen und Ganzen die wichtigsten Ergebnisse dieses Artikels zusammen. Das Titelblatt des GEO-Heftes ist also irreführend. Zwar ist der Untergang der deutschen Sprache mit einem Fragezeichen versehen, aber die einzelnen Beispiele gehören zum bekannten Verfallsrepertoire. Ob die Sprachverfalls-Titelseite als Blickfänger, Ankaufsreiz oder provokante Frage gemeint ist, muss offen bleiben.

2.3 Bereits im GEO-Artikel (Romberg 2012, S. 143) erwähnt ist eine Aussendung der Deutschen Presse-Agentur mit dem als „Rechtschreibrat[s]Chef" bezeichneten Hans Zehetmair. Konkreter Bezug ist wahrscheinlich das Gespräch am 21. Dezember 2012 unter dem Titel „Twitter und ,HDL': Rechtschreibrats-Chef schlägt Alarm“. Ein weiteres Gespräch fand am 
2. Jänner 2012 statt, der Titel der Aussendung ist „Rechtschreibrat-Chef Zehetmair: Twitter und SMS schaden der Sprache“. Im ersten Gespräch fiel das immer wieder zitierte Wort „Fetzenliteratur“, also angeblich durch SMS und Twitter beförderte Sprache in Satzfragmenten; dazu kommen noch die Kritik an Ausdrücken wie super und geil, eine Klage über die mangelhafte sprachliche Qualität von Hochschularbeiten und über die zunehmenden Anglizismen. Im zweiten Gespräch wird der gleiche Themenkomplex diskutiert, ergänzt durch den Ausdruck „Recycling-Sprache“, durch Klagen über mangelnde sprachliche Authentizität und durch kulturkritische Bemerkungen. Als Hochwertwörter werden Begriffe wie „Gefühl“ und „Herzlichkeit" vorgebracht, die „Sprache als Schmuckstück, mit Adjektiven verziert", und als allgemeine Empfehlung: Kinder sollen wieder mehr Gedichte lernen und Bücher lesen, um die Schönheit der Sprache zu erleben. Dazu werden die Eltern in die Pflicht genommen, auch sie selbst müssen lesen, nicht (nur) die Bild-Zeitung. Im GEO-Artikel (Romberg 2012, S. 143) wird die Sachlage ins rechte Licht gerückt: „Keine Sorge, sagt Peter Schlobinski, Experte für Medienlinguistik in Hannover.“ Doch um diese Sachlage soll es hier nicht gehen: Die wichtigsten Informationen finden sich in Einführungsbüchern wie Neuland (2008, Kap. 1) und in den Bänden 7 und 12 der Reihe „Thema Deutsch“ (Schlobinski (Hg.) 2006; Neuland (Hg.) 2012), die Sachlage selbst hat Spitzmüller (2006, S. 42 ff.) prägnant zusammengefasst. Kurz: Fetzenliterarische Erscheinungen kommen aus substandardsprachlichen Varietäten mit register- und domänenspezifischer Funktionalität. Diese Funktionsbereiche werden normalerweise nicht überschritten, es sei denn in den bekannten Fällen wie Zitat und funktionalstilistischer Gebrauch (dazu gehört auch bewusste Provokation). Dass Jugendliche auf mediale Ressourcen zurückgreifen und sie spielerisch verarbeiten (Dürscheid 2006, S.119; Neuland 2011), steht außer Frage. Dabei kann es durchaus auch zu Kollisionen der Werthorizonte kommen (Volmert 2006, S. 92), besonders im Schulalltag. Jugendsprache ist aber hauptsächlich ein Medium der Freizeitkommunikation in jugendlichen Peer-Groups, verbunden mit „mannigfache[n] Friktionen, Konflikte[n] und Widersprüche[n] für die Jugendlichen, aber auch für die Bildungsinstitutionen" (Neuland 2011, S. 22). Traditionelle Normvorstellungen können hinterfragt werden; sie müssen aber in der konkreten Kommunikationssituation ausverhandelt werden können - je besser das gelingt, desto weniger besteht Anlass zur Sorge, und man könnte die ganze mediale Aufregung in sprachwissenschaftlicher Sicht auf sich beruhen lassen. Erstaunlich ist vielmehr, dass diese Meldung schon am 22. Dezember 2012 in der gesamten österreichischen überregionalen Qualitätspresse übernommen wurde. Am Wortlaut der dpa-Aussendung wurde fast nichts geändert und nur wenig gekürzt. Doch der Status von Hans Zehetmair wurde deutlich angehoben, in der „Presse“ (22.12.2012, S. 29) vom 
Rechtschreibrat-Chef zu einem „Rechtschreibexperten“ und im Inneren des Artikels zu einem „Sprachexperten“, in der „Wiener Zeitung“ (22./ 23.12.2012, S. 31) sogar zu einem „Experten“ allgemeiner Art. Nur der „Standard“ (22./23.12.2012) begnügt sich mit der korrekten Benennung in Form des Kompositums „Rechtschreibratsvorsitzender“. Vorsichtig formulierte Kritik wagt nur die „Presse“: „Dabei verfällt der Sprachexperte in ein bekanntes Lamento: [...]." Doch diese Kritik wird nicht weiter ausgeführt.

\section{Sprachkritik ohne Sprache 2: Der Mythos von der deutschen Zeitenfolgeregel}

3.1 Als Sprachkritik ohne Sprache kann man auch den Vorwurf verstehen, es werde - in der deutschen Sprache! - gegen die Zeitenfolgeregeln verstoßen bzw. die Kenntnis dieser Zeitenfolgeregeln sei zunehmend im Schwinden oder sogar nicht mehr vorhanden, wobei dieser Vorwurf meist gegen die „üblichen Verdächtigen“ gerichtet wird, also Jugendliche, Zeitungsleute usw. In diesem Fall ist die Sachlage komplex, sodass ich sie hier nur in Grundzügen erläutern kann. Als Muster für die Zeitenfolgeregel gilt die lateinische Schulgrammatik, hier am Beispiel der Schulgrammatik von Gaar/ Schuster (o.J.). Dort wird die Zeitenfolge meist bei den konjunktivischen Nebensätzen dargestellt, also bei Objektsätzen nach Verben des Fragens, Wissens usw. Die Zeitverhältnisse gleichzeitig - vorzeitig - nachzeitig werden von der Handlung des Nebensatzes aus eingeteilt:

\begin{tabular}{|l|l|l|l|}
\hline $\begin{array}{l}\text { Haupthandlung: } \\
\text { Zeitstufe }\end{array}$ & $\begin{array}{l}\text { 1. Gegenwart } \\
\text { (Zukunft) }\end{array}$ & 2. Vergangenheit & $\begin{array}{l}\text { Nebenhandlung: } \\
\text { Zeitverhältnis }\end{array}$ \\
\hline $\begin{array}{l}\text { Nebenhandlung: } \\
\text { Tempus }\end{array}$ & Präsens & Imperfekt & gleichzeitig \\
\cline { 2 - 4 } & Perfekt & Plusquamperfekt & vorzeitig \\
\cline { 2 - 4 } & $\begin{array}{l}\text {-urus sim } \\
\text { (,umschreibendes Futur“ } \\
\text { bei bevorstehender oder } \\
\text { beabsichtigter Handlung) }\end{array}$ & -urus essem & nachzeitig \\
\hline
\end{tabular}

Die Konjunktive der Nebenhandlung erscheinen so „bei vergangener Haupthandlung um eine Stufe nach der Vergangenheit hin verschoben“" (Gaar/ Schuster o.J., S. 187; Hervorhebungen getilgt). Eine Sonderregel gibt es für das umschreibende Futur. „Das Tempus indikativischer Nebensätze ist freier. Oft haben Nebensätze das Tempus des entsprechenden unabhängigen Satzes" (ebd., S. 189). In der wissenschaftlichen Literatur liest man es anders. Aufschlussreich ist ein genaueres Zitat aus Kühner/Stegmann (1976, S. 174): 
Die Tempora des Konjunktivs haben an sich in Nebensätzen, entsprechend der Entwicklung der Hypotaxe aus der Parataxe, durchaus dieselbe Bedeutung wie in Hauptsätzen; daher wird das Tempus des Nebensatzes von Haus aus nicht durch mechanische Tempusfolge bestimmt, wie man früher wohl angenommen hat, sondern durch Wesen und Inhalt des Nebensatzes. Namentlich neben einem Tempus der Gegenwart oder Zukunft im Hauptsatze stehen fast durchweg dieselben Tempora, die auch im selbständigen Hauptsatze stehen würden [...]. Anderseits lässt sich nicht leugnen, dass sich mit der Zeit gewisse feste Typen der Zeitfolge herausgebildet haben [...]. Dadurch bildete sich, besonders seit der klassischen Zeit, eine gewisse Regelmäßigkeit der Zeitfolge heraus, die den Schriftsteller ungewöhnliche Verbindungen verschiedenartiger Tempora nach Möglichkeit vermeiden ließ; aber anderseits zeigen die zahlreichen Abweichungen von der Hauptregel [...], dass die Wahl des Tempus im untergeordneten Satze in erster Linie immer noch nach dessen Bedeutung entschieden wurde.

Die schulgrammatische Zeitenfolgeregel im Lateinischen ist im Wesentlichen das Ergebnis einer literatursprachlichen Normierung. Die Perfektund Plusquamperfektverschiebung bei Vorzeitigkeit ist durch die ,punktuelle Aktionsart des Perfektstammes"verständlich, worauf Gaar/Schuster (o.J., S. 188, Anm. 7) verweisen. Sie bieten an der gleichen Stelle sogar den Ansatz einer Erklärung an: Angleichung der Zeitstufen von Haupt- und Nebenhandlung als „straffe Durchführung der Unterordnung“ im Einklang mit der traditionellen (heute differenziert gesehenen) Auffassung der Entstehung von Hypotaxe aus Parataxe. Im Grund ist diese Erklärung auch heute noch durchaus vertretbar: Subordination ergibt sich durch den Verlust der syntaktischen Autonomie.

3.2 Umsomehr fraglich sind alle Versuche, eine dem Lateinischen vergleichbare Zeitenfolgeregel im Deutschen (Konjunktiv I nach Präsens, Konjunktiv II nach Vergangenheit) nachzuweisen. Das gilt schon für die ältesten Sprachstufen des Deutschen, wo die Beleglage durchaus kontrovers diskutiert wird (zusammenfassend Schrodt 2007). Alle Versuche, eine solche Zeitenfolgeregel nachzuweisen, müssen mit den schon von Kühner/Stegmann so formulierten ,zahlreichen Abweichungen von der Hauptregel“ ihren Frieden schließen. Dass sich in den Grammatiken der frühen Neuzeit dem Lateinischen angenäherte Zeitenfolgeregeln finden, überrascht nicht; die Sprachwirklichkeit mag schon damals anders ausgesehen haben. Spätestens ab dem 17. Jahrhundert tritt die Zeitenfolgeregel zunehmend außer Kraft, was Elspaß (2005, S. 243 ff.) anhand seines Briefkorpus nachweisen konnte. Auch für die Sprache der Gegenwart wurden schon bald Zweifel an der strengen Zeitenfolge-Regel angemeldet (Gelhaus 1972, S. 29 ff.). Gelhaus (1974) formuliert aufgrund seiner Untersuchungen folgendes Ergebnis, das auch heute noch im Wesentlichen Gültigkeit hat: Es gibt Satzgefüge mit freier Tempuswahl (Einbettungen von Subjektsätzen, Objektsätzen, Kausalsätzen, Konzessivsätzen und Schlussfolgerungssätzen als eigener Typus von 
Konditionalsätzen) und Satzgefüge mit eingeschränkter Tempuswahl (Temporalsätze und Bedingungssätze im engeren Sinn). Die Beschränkung ergibt sich daraus, dass die Tempora von Haupt- und Nebensatz bezüglich des Merkmals „zum Sprechzeitpunkt abgeschlossen“ gleich (positiv oder negativ) markiert sind. Nicht akzeptabel sind also Kombinationen wie *Wäbrend er arbeitete, raucht er nicht und $*$ Hans hatte gearbeitet, während Peter schlafen wird (ebd., S. 64 f.). Offensichtlich kann aber auch das Merkmal der Abgeschlossenheit durch die Handlungsqualität eines der Teilsätze auch für den anderen Teilsatz bestimmt sein. Das zeigt sich in Kombinationen wie PräsensPerfekt Beim Abscbied bält Rosalie zunächst noch den Blick auf ibre Tocbter gericbtet, während sie schon den jungen Mann am Obrläppchen gefasst hat. Im Temporalsatz bezeichnet das Perfekt eine abgeschlossene Handlung; das Geschehen des Hauptsatzes ist vom Tempus aus gesehen zunächst nicht abgeschlossen, wird aber durch den Temporalsatz auf einen punktuellen Ausschnitt eines $\mathrm{Zu}$ stands bezogen. Ähnlich verhält es sich auch mit Sätzen wie Während Hans noch überlegt, hat Peter schon einen Entschluss gefasst / wird Peter schon einen Entschluss gefasst haben. Die Abgeschlossenheit eines Verbalvorgangs kann also nicht nur durch das verbale Tempus bezeichnet sein, sondern auch durch Sinnbeziehungen innerhalb des Satzgefüges. Auch andere aspektanzeigende Ausdrucksformen wie beim Abschied und zunächst noch im oben zitierten Beispielsatz mögen zur aspektuellen Festlegung des Zeitbezugs mitwirken. So ist es auch verständlich, dass Gelhaus (ebd., S. 69) Sätze wie Hans hat Klavier gespielt / wird Klavier gespielt haben, während Peter schlief und Wäbrend sie sich über die Stirn strich, hat sie gesenfit / wird sie geseufżt baben als grammatisch ansieht. Berücksichtigt man alle diese Erscheinungen, so ergeben sich daraus noch zusätzliche Fälle von möglichen Tempuskombinationen auch bei Temporalsatzgefügen. Ausgeschlossen bleiben dann nur noch Sätze, in denen die Zeitbeziehungen sachlogisch nicht stimmen. Das sind Sätze, wie sie auch in der Duden-Grammatik als nicht akzeptabel angeführt sind, wie *Wir spielen Skat, sooft wir uns trafen / getroffen haben / getroffen batten (Duden 2009, S. 516 [ $\$$ 747]). Gelegentlich wird tatsächlich die consecutio temporum im Deutschen nur auf Temporalsatzgefüge bezogen (Wierzbicka/Schlegel 2008). Das hat aber mit einer consecutio temporum nach dem lateinischen Schema nichts zu tun, sondern hier ist einfach ein referenziell falsches Tempus gesetzt. Vergleichbare Erscheinungen gibt es übrigens auch im Spanischen und ebenso einen vergleichbaren Mythos (Kowal 2007).

3.3 Aspektübereinstimmungen sind auch außerhalb von Temporalsatzgefügen vorhanden (Schrodt 1998), nämlich in Objektsätzen, die in einem durch die Semantik des Hauptsatzverbs definierten Aspektverhältnis zum Hauptsatz stehen. Das wird besonders bei faktiven Verben deutlich. So ist in der deutschen literatursprachlich ausgerichteten Standardsprache das Perfekt bzw. das Plusquamperfekt deutlich besser als das Präteritum in: 
(1) Hans bedauert, dass er sein Geld vergessen hat.

(2) Hans bedauerte, dass er sein Geld vergessen hatte.

Die Referenzzeit des Perfekts ist in (1) die Sprechzeit, die des Plusquamperfekts in (2) ein Zeitabschnitt innerhalb des vom Hauptsatz bezeichneten Geschehens. Die Handlung des Nebensatzes ist gewissermaßen ein momenthafter Ausschnitt aus der Handlung des Hauptsatzes; man kann das noch deutlicher durch Ausdrücke wie die (jetzt bqu. damals vorhandene) Tatsache hervorheben. Wenn die Referenzzeit durch Temporaladverbien verlagert oder aufgehoben wird, steht das Präteritum:

(1') Hans bedauert, dass er damals sein Geld vergaß.

Der Objektsatz in (1') bezieht sich auf ein vergangenes Ereignis, das zum Sprechzeitpunkt nur mehr erinnert wird. Ganz ähnlich verhält es sich bei anderen faktiven Verben wie sich ärgern, sich fremen usw. Einige weitere Beispiele:

(3) Hans weiß, dass Peter den Hund geschlagen hat.

(4) Hans weiß, dass Peter zum Vortrag gekommen ist.

In beiden Fällen ist das Präteritum zweifellos ebenfalls möglich, nur würde man dann die Sätze eher so verstehen, dass es sich um längst vergangene, nur mehr erinnerte Geschehnisse handelt, während sich die Formen im Perfekt auf aktuelle Tatsachen beziehen. Bei Hauptsatzverben, die hingegen kein Aspektverhältnis zum Objektsatz festlegen, ist auch die Tempussetzung im Objektsatz frei:

(5) Hans sagt, dass Peter den Hund schlug / geschlagen hat.

Das Perfekt bezeichnet hier keinen momenthaften Ausschnitt aus der Verbalhandlung des Hauptsatzes, sondern es ist dem Präteritum im Wesentlichen synonym und mit ihm austauschbar (mit den bekannten stilistischen Effekten). Man kann das so verstehen, dass hier durch das Perfekt keine zusätzliche Referenzzeit eingeführt wird - oder, wenn man auch für das aktualisierende Perfekt eine Referenzzeit annimmt, dass diese Referenzzeit vom Hauptsatzverb nicht festgelegt wird. Eine zusätzliche Referenzzeit wird erst durch das Plusquamperfekt eingeführt, und zwar obligatorisch. Setzt man (5) ins Präteritum, ergibt sich:

(5’) Hans sagte, dass Peter den Hund schlug / geschlagen hat.

Ein Objektsatz im Plusquamperfekt wäre hier ungrammatisch. Er ist erst dann möglich, wenn eine zusätzliche Geschehensebene als Referenzzeitraum eingeführt wird:

(6) Hans sagte, dass Peter den Hund geschlagen hatte, bevor Ilse ins Haus kam. 
Das, was man hier als Zeitenfolge beschreiben könnte, sollte man eher Aspektkongruenz nennen. Das entspricht dem grammatischen Substrat, der Übereinstimmung mit der Referenzzeit, deutlich besser - und stellt auf diese Weise wieder eine Verbindung zur lateinischen Zeitenfolgeregel her. Mit der schulgrammatischen Zeitenfolge hat das aber nichts zu tun. So sieht das auch schon die ältere deutsche Schulgrammatik, wie etwa Wollmann (1967, \276, ohne Hervorhebungen): „Das noch im Mhd. geltende Gesetz der Zeitenfolge, das im Lateinischen (consecutio temporum), Französischen (concordance des temps) und Englischen (sequence of tenses) eine wichtige Rolle spielt, hat im Nhd. keine Geltung mehr." Auch stärker normbezogene Gebrauchsgrammatiken wie Jung (1980, S. 235 f.) beschränken die „strenge“ Zeitenfolge auf konditionale Satzgefüge (Wenn ich Zeit babe, komme ich. - Wenn ich Zeit bätte, käme ich. - Hätte ich Zeit gehabt, so wäre ich gekommen.). Doch auch hier könnte man fragen, ob Konjunktiv II und Konjunktiv II Perfekt obligatorisch sind. Eine Art Zeitenfolge beschreibt Jung (1980, S. 212f.) unter dem Begriff „relativer Gebrauch der Tempora“. Aber es gibt vergleichbare Regeln nur bei Vorzeitigkeit, und auch da ist es fraglich, ob nicht auch andere Tempora sinngemäß gesetzt werden können. Ein weiteres Problem ist die Unterschiedlichkeit des Nebensatztyps: Objektsätze, Konditionalsätze, Temporalsätze und Relativsätze werden zusammen dargestellt. Der Normalfall ist jedenfalls, dass das Tempus im Nebensatz die gleiche Bedeutung hat wie im Hauptsatz. Daher ist eine genauere grammatische Darstellung der Zeitbezüge in Temporalsätzen nicht sehr sinnvoll. Das scheint auch Ulrich Engel erkannt zu haben: In der 1. Auflage seiner „Deutschen Grammatik" (1988) werden die Zeitbezüge im Abschnitt S 116, S. 260 ff., auf sechs Seiten zusammengefasst, in der Neubearbeitung (2004) ist dieser Abschnitt ausgelassen. Engel (2004, S. 267 f.) sieht in den deutschen Tempusformen drei semantische Bereiche bezeichnet: Wirklichkeit, Modalität und Zeit, und wendet sich damit ausdrücklich gegen ein „System“ von sechs Tempora. Seiner Ansicht nach ist dieses „System“ ein Popanz, den es zu entlarven gilt und mit dem sich nach der Entlarvung wohl leben lässt - das könnte man ohne Abstriche auch für die deutsche Zeitenfolgeregel behaupten.

3.4 Dieser Popanz ist aber in anderen Zusammenhängen noch durchaus lebendig. Für grammatische Zweifelsfälle empfiehlt sich der Duden-Band „Richtiges und gutes Deutsch“ (Duden 2002). Dort (S. 964) ist etwas kryptisch vermerkt: „Unter Zeitenfolge (Consecutio Temporum) versteht man das Verhältnis der Zeiten in einem Satz. Die Zeitenfolge gilt nur für die Formen des Indikativs; im Bereich des Konjunktivs ist das System weitgehend gestört." Es gibt also doch ein System, und es kann sogar gestört werden (oder stört es sich selbst?). Von einer solchen Formulierung ist es nicht weit, tatsächliche Störer ausfindig zu machen: Es sind die üblichen Verdächtigen, besonders die jungen Menschen, die im Grammatikunterricht nicht 
recht aufgepasst haben. Das ist einem übrigens sehr sorgfältigen und umsichtigen Projektbericht von Markus Rheindorf (2011) ,Wandel der Deutschen Sprache: Eine textsortenbezogene Pilotstudie“, der im Internet verfügbar ist, zu entnehmen. Dieser Projektbericht liegt in zwei Fassungen vor. Die erste Fassung (Rheindorf 1) mit dem Internet-Titel „WandelderSprachePilotstudie" enthält 72 Textseiten, die zweite Fassung (Rheindorf 2) mit dem Titel „Pilotstudie_Sprachwandel“ ist etwas erweitert und enthält 77 Textseiten. In beiden Fällen ist das Datum des Projektberichts mit „31.5.2011“ angegeben, bei Rheindorf 2 mit der Anmerkung „Einige Erläuterungen zwecks allgemeiner Verständlichkeit hinzugefügt, April 2012“. Es geht um Sprachfehler in schriftlichen Reifeprüfungen, erhoben aus einem kleinen, aber durchaus repräsentativen Korpus von Maturaarbeiten aus Graz und Wien. In Rheindorf 1, S. 76, liest man den allgemeinen Hinweis: „Die deutsche Syntax hat als Fehlerquelle leicht zugenommen, hierbei sind wie bereits erwähnt vermehrt Tempus und Zeitenfolge, die nicht immer vollständig beherrscht werden.“ Rheindorf 1, S. 32 f.: „In den Texten von 1970 finden sich in diesem Bereich keine Normverletzungen, 2010 sind es immerhin 12 Fälle (3 davon in Graz, 9 in Wien), in denen die korrekte Zeitenfolge nicht eingehalten wird." Rheindorf 2 (S. 36) hat offensichtlich auf meine per Mail vorgetragene Kritik reagiert und verwendet nun den Begriff „Aspektkongruenz“, meint aber noch immer dasselbe. Nun sind Beispiele angeführt (meine Zählung):

(7) Es war früher so, dass man erst nach der Geburt erfährt, ob das Kind krank oder gesund ist.

(8) Man begann gerade Fortschritte zu machen, da hatte ein tragischer Zwischenfall alles zunichte gemacht.

(9) Die Entscheidung, was ich in meinem späteren Berufsleben tun wollte, musste ich also schon vor dem Eintritt in die Oberstufe getroffen haben.

(10) In der Erzählung begegnen sich ein Mann und eine Frau, verbringen die Nacht zusammen, ohne sich gekannt zu haben.

(11) Während wir uns damit beschäftigt haben, verstanden wir die Bedeutung der Stammzellenforschung für die Medizin.

Also gibt es doch eine Zeitenfolge? Ich sehe es anders: In allen Fällen ist eine autonome Tempussetzung naheliegend. (7) enthält im Objektsatz ein generelles Präsens: Die Erfahrung nach der Geburt ist nicht etwas, das auf die Handlungszeit des Trägersatzes beschränkt ist. In (8) kann das Plusquamperfekt eine besondere modale (adversative) Bedeutungskomponente bezeichnen (Wunderlich 1970, S. 151 f.). In (9) würde man eher einen präsentischen Relativsatz erwarten, doch hier dient das Erzähler-Jetzt als zeitlicher Fixpunkt, und das ist auch nach der neuen Duden-Grammatik (Duden 2009, S. 515 [\$ 747]) korrekt. (10) ist überhaupt korrekt: Wenn eine Frau und ein Mann zusammen die Nacht verbringen, müssen sie sich schon dadurch 
kennen und wohl auch schon wenigstens kurz vor ihrer nächtlichen Aktion gekannt haben. Die gegenseitige Unkenntnis ist in dieser fraglichen Nacht ganz eindeutig vergangen. (11) wird besser, wenn man statt verstanden das Verb in erkannten ändert: Die Beschäftigung führt zur Erkenntnis, aber vielleicht auch zum Verstehen. Ungewöhnlich mag das Perfekt im Temporalsatz erscheinen, aber die Handlung ist eindeutig nicht resultativ (präteritales Perfekt). Ich sehe in diesen Beispielsätzen keine Grammatikfehler, sondern einzelne stilistische Ungeschicklichkeiten.

3.5 Der lebendige Popanz zeigt sich besonders deutlich, wenn man im COSMAS-Korpus des Instituts für Deutsche Sprache mit dem Stichwort „Zeitenfolge“" nach Belegen sucht. Dort ist nicht nur der grammatische Terminus gemeint; wenn das aber der Fall ist, dann ist in den Zeitungsbelegen meistens ein sprachkritischer Ton zu hören. Einige Beispiele:

„Bevor unsere Mutter starb“, steht da in einer beschreibenden Passage, „stellte sie ein Video zusammen, von dem sie annahm, dass es Lisa vielleicht gefällt.“ „Aha“, denken wir, „keine Zeitenfolge, kein Konjunktiv. Vielleicht hält sich auch Sedaris in dieser Passage nicht an die Regeln der Grammatik?" (Zürcher Tagesanzeiger, 17.5.1999, S. 55)

Die Sprache ist blaß, auf die Zeitenfolge wird kein Wert gelegt; der alberne Diktierstil, der freier Erzählweise widerrät, bremst die Bereitschaft mitzuempfinden, gelegentlich nachgeschobener Gefühlsschwulst wirkt eher aufgetragen und peinlich. (Nürnberger Nachrichten, 5.10.1991, S. 22; über ein Buch von Ulrich Sahm)

»Ich bin zuversichtlich, dass am Ende des heutigen Tages eine Verständigung erzielt wurde«, sagte SPD-Innenpolitiker Dieter Wiefelspütz in nicht ganz korrekter Zeitenfolge. (Nürnberger Nachrichten, 26.5.2004)

Zeitenfolge nicht im Griff

Zum Thema Grammatik

Nein, ich mache bestimmt nicht alles richtig, sonst brauchte ich keine ganze Dudenreihe zum Nachsehen. Sixtus Beckmesser ist auch nicht meine Lieblingsfigur in den Meistersingern, dennoch stößt es mir sauer auf, wie oft gestandene Redakteure beiderlei Geschlechts die deutsche Grammatik verunstalten.

Gerade heute habe ich wieder ein Paradebeispiel gelesen, einen Bericht, in dem entweder Wirkung und Ursache vertauscht wurden oder beim Abfassen der Zeitdruck zu groß war - oder die Verfasserin mit Imperfekt und Plusquamperfekt (auf deutsch der 1. und der 3. Vergangenheit) auf Kriegsfuß steht. (Nürnberger Zeitung, 20.5.2011, S. 5; Zeitenfolge nicht im Griff)

*Zu: „Zeitenfolge nicht im Griff“ vom 20.5.

Vielen Dank für den Leserbrief „Grammatik“. Ja, unsere Sprache wird grausam verschandelt. Einfachstes wird falsch gemacht. Es fehlen Endungen $(, \ldots .$. mit dem Herz...“ und ,...was beim Mensch hilft...“), es wird falsch gebeugt (,....giltet das nicht..." und ,abgewägt"). Bei ,gewinkt" gibt der Duden jetzt schon nach! Frau Britt im Fernsehen entschuldigte sich kürzlich, weil sie gesagt hatte: „Ihr trenntet Euch!“ und das als falsch empfand. Die Mehrzahl wird falsch gebildet (,Zu- 
wächse"). Sehr gut, dass Herr Jauch es vor ein paar Tagen wagte, eine Kandidatin zu verbessern, weil sie eine Endung weggelassen hatte. (Nürnberger Zeitung, 10.6.2011, S. 6; Lesermeinungen kurz gefasst)

„Wir haben nun starke Beweise, daß der Irak mit einem großen Programm zur Entwicklung von biologischen Waffen für Angriffszwecke beschäftigt ist." So hörte es das aufmerksam lauschende Publikum bei einem Christopher-Vortrag vor der jüdischen Anti-Defamation-League, und so wurde es auch auf das Tonband gebannt. In seinem Redetext jedoch lag die verbotene Beschäftigung des Irak bereits in der Vergangenheit. Las der Minister falsch vom Blatt? Ging er absichtlich vom vorbereiteten Text ab? Oder hat er gar Probleme mit der Zeitenfolge? Lauter Fragen, die mitunter ebenso schwer zu beantworten sind, wie die nach den wahren Ambitionen des Irak. (Die Presse, 6.4.1995, Ressort: Ausland)

Besonders ergiebig sind die Diskussionsbeiträge in der Wikipedia (unter http://de.wikipedia.org/wiki/Diskussion):

Wer sollen denn jetzt „sie“ sein? „Jesusfreund [...] mit seinen Deutschkentnissen“ bekommt keine gute B-Note für Zeitenfolge. (Benno_Ohnesorg/Archiv/2009: Wikipedia, 2011)

Es wäre überdies nett, wenn IP 85.178.23.205 unterschreiben und die deutsche Grammatik (Zeitenfolge) etwas sorgfältiger beachten würde. (Cuba_Sí: Wikipedia, 2011)

„Nachdem seine Schüsse den davontreibenden Blake verfehlen, erschießen er und Nobody" - erschossen hätten sie einander mit oder ohne Treffer auf Blake. Es geht nur um eine Reihenfolge von Fakten. Das verwischt die Verwendung von „nachdem“. (Die Zeitenfolge wäre übrigens: „Nachdem... verfehlt haben, erschieBen“.) (Dead_Man/Archiv: Wikipedia, 2011)

... schien es nur zu wahrscheinlich, dass Genêt unter der Guillotine hingerichtet werden würde, würde er den Jakobinern übergeben. Stimmt da die Zeitenfolge? Wäre nicht ...wäre er den Jakobinern übergeben worden die richtige Grammatik? (Edmond-Charles_Genêt: Wikipedia, 2011)

c) der Part über seine AIDS-Erkrankung wirkt wirr und widersprüchlich (falsche Zeitenfolge, zu häufige Sprünge). (Freddie_Mercury/Archiv/1: Wikipedia, 2011)

\section{Zeitenfolge}

Hat jemand etwas dagegen, wenn ich im Abschnitt zur Gründungsgeschichte die korrekten (Verb-)Tempi einarbeite? Da ich mit diesem Artikel nicht verheiratet bin, frage ich mal lieber an. Gruß F. (Lehmbruck-Museum: Wikipedia, 2011)

Satzunglücke wie „Wäre ein Amateurklub, von dem die Profimannschaft in der Bundesliga spielt, auf Platz Eins gestanden, wäre der Zweitplatzierte aufgestiegen, außer das Profiteam dieser Mannschaft würde den Abstiegsplatz der Bundesliga belegen." Zeitenfolge, Ausdruck, Schachtelung und Beziehungsfehler. - Mannschaft und Verein sind bei Zeiten eben keine Synonyme - in dieser Dichte verbieten ein Babberl. Allgemein weiss der Artikel nicht, ob er eine gram. Vergangenheit oder historisches Präsens nutzen will, sondern wechselt teilweise bis zur unfreiwilligen Komik - die Konjunktive wechseln an einzelnen Stellen in einem Satz von Gegenwart in Vergangenheit und zurück. Aber selbst da, wo die Sprache nicht 
vollkommen daneben geht, bleibt immer noch Fansprech für Fans. Ist zwar ein großes Problem aller Sportartikel, aber bei den soliden Babberlsportartikel besser gelöst und das kann man auch verlangen. (Österreichische_Fußballmeisterschaft_ 2008/09: Wikipedia, 2011)

Logischer Fehler

Hallo, wenn ich diesen Satz lese bekomme ich einen Knoten in der Zeitenfolge: „Zusammen mit seiner Frau arbeitet Krugman zur Zeit an einem Lehrbuch über Mikro- und Makroökonomie, das im Dezember 2005 unter dem Titel Economics erschien.“ (Paul_Krugman: Wikipedia, 2011)

Ich kann hier die einzelnen Stellen nicht kommentieren; manche Bemerkungen sind durchaus berechtigt und verweisen auf stilistische Ungeschicklichkeiten. Andererseits zeigen diese Belege, dass die Zeitenfolge auch dort, wo sie noch immer als grammatische Regel gilt, für (wohl nicht professionelle) Autoren Schwierigkeiten macht. Oft wird aber auch die Zeitenfolge unspezifisch im Zusammenhang von abwertenden und inhaltlich-kritischen Bemerkungen gebraucht, gelegentlich auch von Kritikern, die in ihren eigenen Texten fehlerhafte Ausdrücke verwenden (Tempi als Plural von Tempus).

\section{Sprachwandel und Sprachkritik}

4.1 In sprachwissenschaftlicher Sicht könnte das Thema „Sprachkritik“ mit den Argumentationen „Sprachwandel statt Sprachverfall“ und „Sprachgebrauch als Grundlage für Sprachrichtigkeit" erledigt werden. In jedem einzelnen Bereich kann man recht genau erklären, warum es zu Veränderungen kommt und welchen Sinn Änderungen im Formenbereich der Sprache haben können. Es gibt zur Sprachverfallsliteratur auch eine entsprechende wissenschaftliche „Gegenliteratur“. Zu den bekannten Büchern von Bastian Sick (2005, 2006a, 2006b) haben Meinunger (2008) und Ágel (2008) einiges richtiggestellt, und es gibt auch entsprechende übersichtliche und zusammenfassende Darstellungen, z.B. Schrodt (1995). Besonders hervorheben möchte ich hier das überaus informative Buch von Annette Trabold (1993), dem ich eine Neuauflage wünsche. Mit der Laienlinguistik und ihren Erscheinungen befassen sich mehrere Forschungsprojekte, auch am Institut für Deutsche Sprache. Diese „Gegenliteratur" hat ebenfalls eine lange Geschichte: Zu Wustmanns „Allerhand Sprachdummheiten“ (Wustmann 1891) gibt es das Büchlein von Jakob Minor (Minor 1892) „Allerhand Sprachgrobheiten", und auch unter den Sprachratgebern finden sich auch schon ältere Arbeiten, die sich um ein ausgewogenes Urteil bemühen und Sprachwandelerscheinungen berücksichtigen (Vernaleken 1900; Matthias 1929; Sanders 1908). Die aktuellen Zweifelsfälle-Ratgeber (Duden 2002; Wahrig 2009) sind im Großen und Ganzen zuverlässig und berücksichtigen auch neuere Entwicklungen und regionale Erscheinungen. Sogar unter der 
Gebrauchsliteratur gibt es Publikationen, die wenigstens Ansätze von Toleranz gegenüber Sprachveränderungen zeigen (Mackowiak 2008). Doch das Grundproblem des Verhältnisses von Sprachrichtigkeit und Sprachkritik bleibt davon unberührt. Es ist am besten im ersten Buch des RhetorikWerkes von Quintilian aus dem ersten Jahrhundert nach Chr. beschrieben. Quintilian kritisiert Analoge und Etymologie als Grundlagen für die Beurteilung von Sprachrichtigkeit und fragt danach, was nun als argumentative Basis übrig bleiben kann. Seine Bemerkungen sind auch nach Jahrhunderten gültig (nach der Ausgabe Rahn, S. 105):

So bleibt also der Sprachgebrauch noch (zur Betrachtung) übrig; denn es wäre ja fast lächerlich, die Redeweise, wie die Menschen (früher) gesprochen haben, der Redeweise, wie man jetzt spricht, vorzuziehen. Und was ist denn die alte Sprache anderes als der alte Sprachgebrauch? Aber gerade hierfür braucht man Urteilskraft und muss vor allem festsetzen, was wir denn eigentlich Sprachgebrauch nennen. Wenn er seinen Namen nach dem hat, was die Mehrzahl tut, wird er eine höchst gefährliche Vorschrift geben, nicht nur für die Rede, sondern, was mehr bedeutet, für das Leben: Woher nämlich käme das Gute in solcher Fülle, dass der Mehrzahl gefiele, was recht ist? Wie also das Auszupfen der Haare, das Auftürmen von Haarflechten und die Gelage in Bädern, wieweit sie auch im Staat eingedrungen sind, nicht Brauch sein werden, weil nichts von diesen Dingen ohne Tadel bleibt - während wir dagegen nach Brauch baden, das Haar scheren und unsere Festmähler halten -, so wird man auch beim Sprechen nichts als Sprachregel nehmen, wenn es sich in fehlerhafter Weise bei vielen festgesetzt hat. Denn, um zu [dem] überzugehen, wie im Volk die Ungebildeten sprechen, so wissen wir doch, wie oft schon ein ganzes Theaterpublikum und die ganze Menge im Zirkus barbarisch gebrüllt hat. Also werde ich das Gebräuchliche in der Sprache die Übereinstimmung der Gebildeten nennen, so wie im Leben die Übereinstimmung der Guten.

Das Gebräuchliche in der Sprache wird durch die Übereinstimmung der Gebildeten begründet. Die Gebildeten sind im Leben die Guten. Ihre Übereinstimmung sichert die Sprachnormen. Sprache als selbstregulierendes System, das seine Funktionalität im Sprachwandel durch Formenumbau erhält, ist hier nicht im Blick. Dieser Konflikt ist grundsätzlich unauflösbar: So funktioniert Sprachkritik ohne Sprache.

4.2 Die Übereinstimmung der Gebildeten und Guten sichert aber nur dann allgemein verbindliche Sprachnormen, solange das Sozialgefüge der Sprechenden stabil bleibt und solange die Gesellschaft mit ihren gruppenspezifischen Lebensformen im Zusammenwirken sich selbst stabilisiert. Sobald die Grenzen dieser Lebensformen durchlässig werden oder sobald sich die kulturbegründenden Zentren auflösen und damit ihre integrierende Funktion nicht mehr wahrnehmen können, werden andere Sprachgebräuche sichtbar und bilden Material für kommunikative Aufgaben, die in einer stabilen Gesellschaft mit elitären Eigenschaften anders gelöst worden wären oder sich in dieser Form nicht gestellt hätten. Ich zeige das an nur einem Beispiel aus dem Bereich der Jugendsprache (te Neues Verlag (Hg.) o.J., S. 32 f.): 


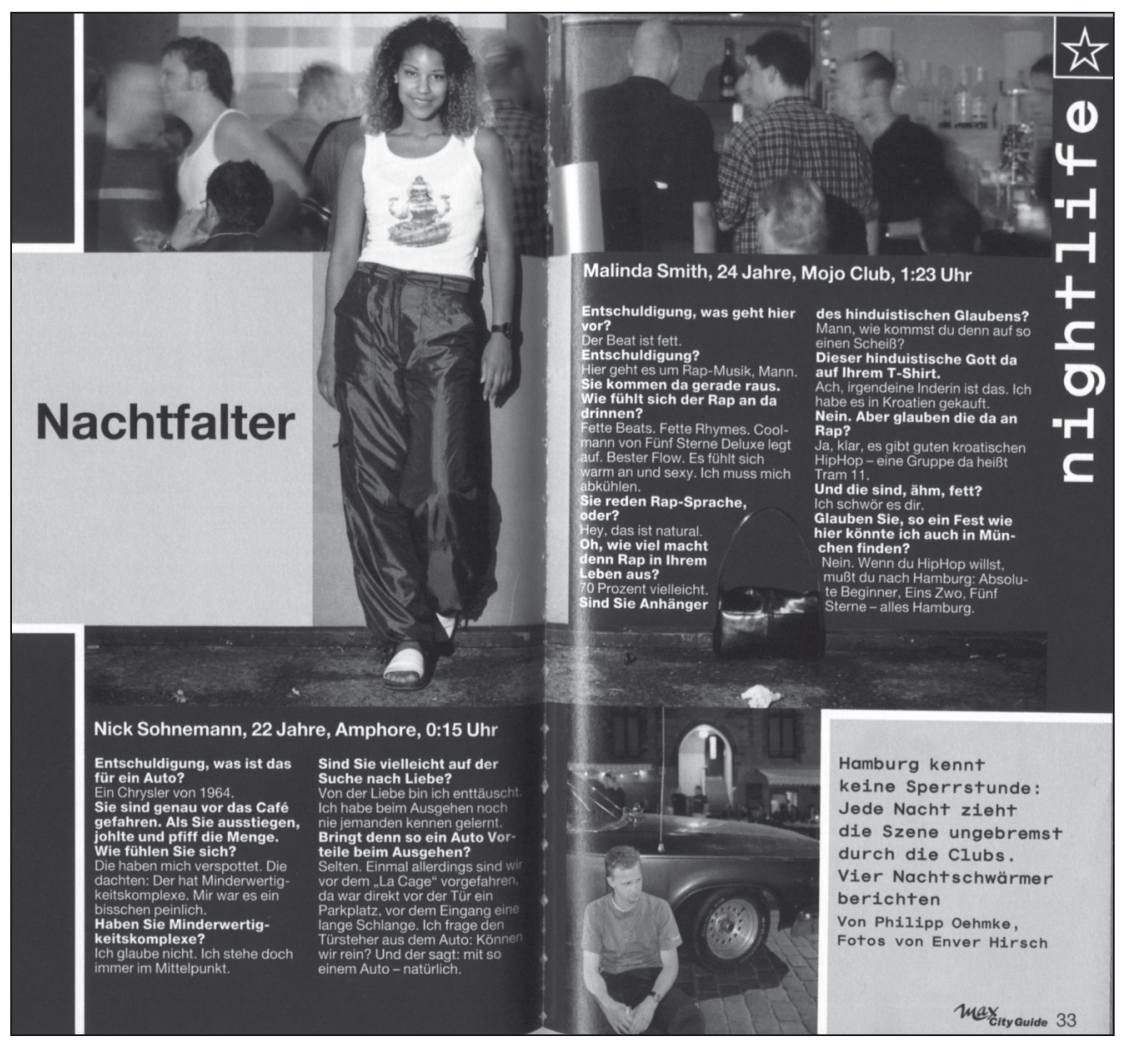

Abb. 1: Rapsprache (Quelle: MAX City Guide Hamburg)

Wir sehen hier ein Beispiel für das, was Spitzmüller (2006, S. 45 f. unter Bezugnahme auf Arbeiten von Neuland und Januschek) einen „diskursiven Wandel“" genannt hat, die Entwicklung eines positiven Bildes von Jugendlichkeit in den 1990er Jahren - ein Bild, das ebenso von Stereotypen geprägt ist wie die sprachkritische Opposition. „Dabei werden Jugendliche und ihre Sprache nicht weniger zur Projektionsfläche gesellschaftlicher Werte als im jugendsprachkritischen Diskurs [...].“ Zeichen dieser „,medialen Überhöhung" finden sich hier in diesem Ausschnitt aus einer Werbeschrift für die Stadt Hamburg deutlich: Die Rap-Sprecherin ist ein adrettes Mädchen mit sehr gemäßigten subkulturellen Merkmalen (Freizeitkleidung, dunkle Hautfarbe, vielleicht auch die Frisur), und sie lächelt sehr freundlich. Die Jugendlichen sind in einem gemäßigten Alter, eigentlich schon junge Erwachsene. Die sprachliche Anarchie ist ebenfalls sehr gemäßigt, und dennoch bildet sich im Text wenigstens andeutungsweise die Differenz der Generationen ab, wenn der Reporter etwas schamhaft fragt Und die sind, ähm, fett? und die Antwort erhält Ich schwör es dir. Die Kollision der Werthorizonte (Volmert 
2006, S. 93) präsentiert sich gemäßigt. Es kommt nicht darauf an, dass die Sprache in dieser Werbeschrift authentisch ist (sie ist es höchstwahrscheinlich nicht). Es kommt nur darauf an, dass sich Formen der Jugendsprache aus der subkulturellen Umgebung herausbewegt haben und - natürlich wiederum domänen- und registerspezifisch - ihren Wert in der Alltagskommunikation gesichert haben.

4.3 Ähnliche Fälle kann man auch in der Kiezsprache (Wiese 2012, dort auch die Beispiele) beobachten. Auch hier sind allgemeine Sprachverfallsklagen fehl am Platz: „Kiezdeutsch ist Teil eines Repertoires, in dem das Standarddeutsche ebenso seinen Platz hat. Sprachkompetenz bedeutet auch, aus diesem sprachlichen Repertoire je nach Situation eine angemessene Wahl zu treffen" (ebd., S. 212 f.). Unter den kiezsprachlichen Wendungen sind Konstruktionen ohne Artikel bekannt - so bekannt, dass sie als besonders auffällige Eigenschaft gelten können. Ein Jugendlicher zu seinem Freund, der bei Rot über die Straße läuft: Machst du rote Ampel!. Über Transportmöglichkeiten: Hast du U-Babn? - Nee, ich hab Fahrrad. Am Einband ist zitiert: Lassma Kino gehn. Wiese (ebd., S. 76 f.) versteht diese Bildungen als neue Funktionsverbgefüge. Ich sehe in solchen Konstruktionen eher neue Inhaltsobjekte, welche die Verbalhandlung unspezifisch charakterisieren. Ihre syntaktische Funktion ist weder die eines Akkusativ- oder Dativobjekts noch die eines Lokalkasus. Solche neuen Objekte finden manchmal ihren Weg in die Tageszeitung. In einem „Leserbrief“ (eigentlich Lesermail) des in Österreich sehr bekannten Lehrers und Autors Niki (Nikolaus) Glattauer wird folgender Dialog zwischen einer Lehrerin und einer etwa 12-jährigen Schülerin zitiert (Kurier, 11.2.2013, S. 15):

- Frau Lehrer, die Sabine fehlt.

- Hab ich gesehen, Merve.

- Sie ist im Park. Sie sagt, sie geht nicht Schule.

- In die.

- Was?

- In die Schule, heißt das. War sie gestern auch im Park?

- Nein, gestern ist sie bei dem Mann gegangen.

- Zu. Zu dem Mann. Du meinst ihren Vater.

- Nein, bei dem anderen Mann.

- Zu. Zu welchem anderen Mann?

- In seine Wohnung. Sie tun fernsehen. Er raucht auch.

(Die Lehrerin ruft besorgt die Sabines Mutter an, kann sie aber nicht erreichen. Zurück in die Klasse fragt sie. wer diesen Mann kennt. Cafer weiß es:)

- Ich glaube, es ist ihr Cousin. Er geht Poly. ${ }^{1}$

- Er geht Poly? Wie alt ist er?

- Eh nicht ganz alt. 16 oder 17.

$1 \quad$ Poly $=$ Polytechnische Schule, eine Zwischenstufe zwischen Pflichtschule und Berufsausbildung. 
Es ist nicht sicher, ob es sich um ein authentisches Beispiel handelt; der Text hat hier wohl auch die Funktion einer Kultur-Comedy (Canoğlu 2012, S. 46 f.). Aber der Autor ist Lehrer und hat Erfahrungen genau in diesem Milieu, das er zu Wort kommen lässt. Solche neuen Inhaltsobjekte sind im grammatischen Bau des Deutschen durchaus funktional: Der Schulgang ist ein komplexes Verhaltensmuster, bei dem die Richtung kommunikativ nicht wichtig ist (die Park-Situation wird korrekt durch eine Präpositionalphrase bezeichnet). Vielleicht ist auch der normabweichende Gebrauch der Präposition bei sachgemäß. Im letzten Teil des Dialogs übernimmt die Lehrerin den substandardsprachlichen Ausdruck: Bei einer Nachfrage wäre eine Korrektur nicht angemessen, aber vielleicht hat sie schon andere Sorgen als die korrekte Grammatik. Knapp eine Woche danach hat die Kiezsprache schon die erste Seite der Zeitung erobert:

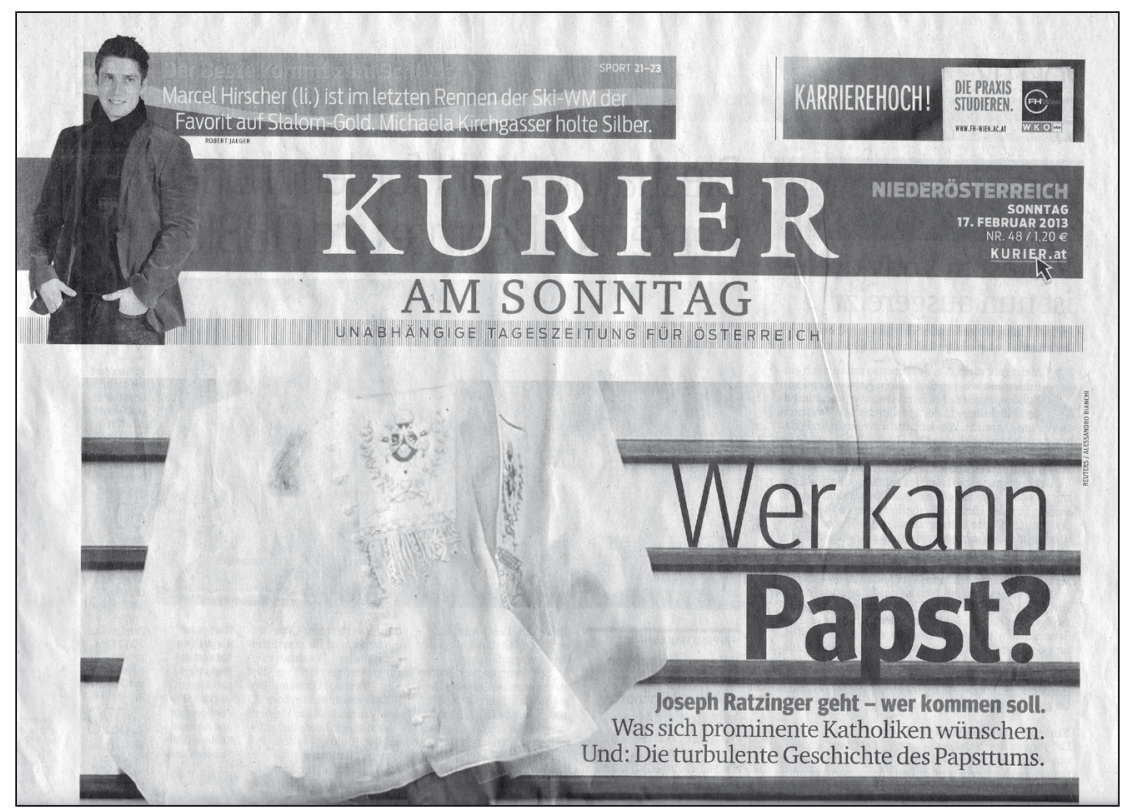

Abb. 2: Kurier (Ausgabe vom 17.2.2013)

Formen der Kiezsprache sind funktionalstilistisch in manchen Kommunikationssituationen durchaus adäquat: Sie sind neu, auffällig, expressiv, oft emotionell, und charakterisieren Einstellungen und Werthaltungen, die im Journalismus und in formloser Kommunikation durchaus wirkungsvoll sein können, kurz: Sie kommt gut an. Der Journalist Dietmar Krug zeichnet ein sympathisches Bild der Kiezsprache in einer Rezension von Wiese (2012): 
Und es ist auch kein gebrochenes Deutsch, wie uns die Medien weismachen wollen, kein radebrechender Ghetto-Slang von Menschen, die es einfach nicht besser können. Es ist eine Sprache mit klaren Regeln in Wortschatz, Satzbau und Grammatik. Und weil dem so ist, wagt Heike Wiese eine These und hebt das Kiezdeutsch in den Rang eines Dialekts, eines „Turbodialekts“, weil er noch so jung und dynamisch ist. Denn in seinen Abweichungen vom Standard folgt das Kiezdeutsch nur der dialektalen Sprachlogik. Ein Beispiel: „Lassma Kino gehen“, sagt man auf Kiezdeutsch. Wenn das falsch ist, weil man schließlich ins Kino geht, dann hat auch jener Steirer falsches Deutsch gesprochen, der unlängst meine Frau gefragt hat: „Bist in Murau Schul gangen?“ Und wenn die kiezdeutsche Ankündigung „Ich frag mein Schwester“ grammatikalisch falsch ist, was war dann die Frage eines alten Bewohners meines rheinischen Heimatdorfes, als er zum ersten Mal meine Frau gesehen hatte und wissen wollte, ob sie zu mir gehört: „Is dat et Dietmar et sin?"“

Naserümpfen löst das Kiezdeutsch ja nicht deshalb aus, weil es wie Kauderwelsch klingt, sondern weil es eine Unterschichts-Jugendsprache ist, deren Aufgabe nun einmal darin besteht, den Sprecher möglichst rotzig von den „Alten“ abzugrenzen. (Die Presse, 10. Februar 2013, S. 10)

So ist es verständlich, dass sich manche kiezdeutsche Formen vielleicht auch über den lexikalischen Bereich hinaus als Innovationen verbreiten können, wobei „[d]ie sprachliche Akkomodation Erwachsener an Jugendliche, die Imitation massenmedialer Stereotype und die Mitnahme jugendsprachlicher Varianten ins Erwachsenenalter [...] als parallel wirksame Prozesse anzunehmen [sind]“ (Androutsopoulos 2005, S. 203). Ihre soziostilistische Markierung wird es aber wohl verhindern, dass sie in den Kernbereich der standardsprachlichen deutschen Syntax vordringen.

4.4 Die sprachgeschichtlichen Vorgänge hat Mattheier (1995, S. 10) so zusammengefasst:

Soziokulturell bedingte Variation entstammt in erster Linie drei verschiedenen Quellen. Einmal entstehen sozio-kommunikative Varianten bei dem Versuch der Anpassung von in der Kompetenz vorliegenden Sprachhandlungsmustern an die jeweils neue Sprachhandlungssituation und -intention. Dann werden, verursacht durch Bewertungsdifferenzen, ständig Varianten aus anderen Sprachvarietäten und auch Sprachen entlehnt. Und schließlich gibt es auch noch die bewusste und geplante Produktion von Sprachvarianten im Zusammenhang mit Sprachplanungsprozessen. Die reale Sprachproduktion ist also durch Variantentypen verschiedenster Provenienz geprägt. Die in der Sprache vorhandenen Varianten bilden nun das Reservoir, aus dem sich einige dieser Varianten als Innovationen herausbilden, d.h. als Ansatzpunkt für dauerhafte Veränderungen. 


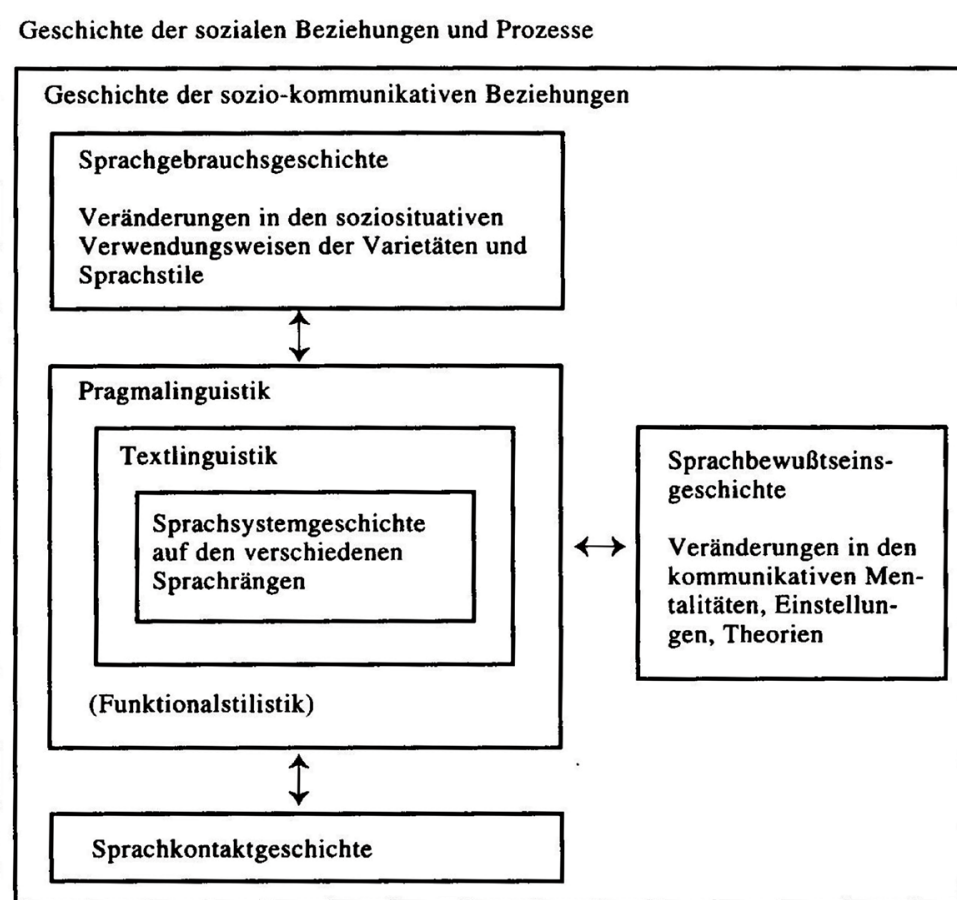

Abb. 3: Quellen der sprachkulturellen Variation (Mattheier 1995, S. 15)

\section{Von den Kräften der deutschen Sprachkritik}

5.1 Sprachkritik ohne Sprache ist aber keineswegs eine paradoxe Erscheinung: Sie belegt eine wichtige Funktionsstelle im öffentlichen und privaten Diskurs über Sprache. Zu ihrer Erklärung eignet sich die Begrifflichkeit, die Niklas Luhmann in seiner Theorie sozialer Systeme aufgebaut hat (Übersicht: Luhmann 2012; einige Formulierungen in Anlehnung an Kneer/Nassehi 1997); ich muss mich hier auf einige wenige Bemerkungen beschränken. Gesellschaftliche Ordnung gründet sich auf einen generellen Wertekonsens. Dieser Wertekonsens ist im sozialen Leben fest institutionalisiert und wird von den Handelnden im Sozialisationsprozess verbindlich erworben. Oberster Bezugspunkt der funktionalen Analyse ist die Komplexität der Welt. Für das menschliche Bewusstsein ist die Komplexität der Welt in ihrer Menge alles dessen, was möglich ist, nicht fassbar und erfahrbar; sie überfordert die Fähigkeit des Menschen zu bewusster Erlebnisverarbeitung. So entsteht zwischen der äußersten Weltkomplexität und dem menschlichen Bewusst- 
sein eine Lücke. Hier treten soziale Systeme ein: Sie übernehmen die Aufgabe der Reduktion von Komplexität und bilden „Inseln geringerer Komplexität". Die möglichen Zustände oder Ereignisse werden sozusagen auf ein menschliches Maß verringert und geben dadurch den beteiligten Personen Orientierungshilfen. Damit soziale Systeme funktionieren, müssen sie aber, metonymisch formuliert, stets in einen Diskurs über die Grenze zwischen System und Umwelt eingehen. Diesen Diskurs leistet die Sprachkritik. Sie hat den Status einer Beobachtung und bezeichnet etwas anhand einer Unterscheidung, sagen wir gleich: richtige und falsche Sprache (wobei der Ausdruck „Sprache“ polysem ist - es können Sprachformen, Sprachvorkommnisse, Sprachgebräuche, Sprachbräuche, vielleicht auch Sprachträger sein). Im Rahmen einer Unterscheidung kann aber immer nur eine Seite betrachtet werden, also hier die falsche Sprache. Um falsch und richtig zusammen beobachten zu können, bedarf es einer Beobachtung zweiter Ordnung: Das leistet die (wissenschaftliche) Kritik der Sprachkritik. Durch diese Beobachtung zweiter Ordnung verschiebt sich auch die objektbezügliche Begrifflichkeit: Man wird nicht mehr so einfach von richtig und falsch sprechen wollen, sondern von kommunikativ funktional bzw. nicht funktional. Soziale Systeme haben keinen unmittelbaren Kontakt zu ihrer Umwelt. Die Beobachtung ist eine systeminterne Operation und damit immer eine Konstruktion eines Systems mittels kommunikativer Akte. Eine bestimmte Kommunikation verweist selbstreferenziell auf vorhergehende Kommunikationen (nach Luhmann ,mitlaufende Selbstreferenz"). So wird in sozialen Systemen die Selbstreferenz mit Fremdreferenz angereichert: In operativ geschlossenen Systemen kann so auf Anderes verwiesen und damit Offenheit erzeugt werden. Jede Beobachtung ist an eine spezifische Unterscheidung gebunden. Es gibt immer mehr als eine Unterscheidungsmöglichkeit, in unserem Fall also falsch - richtig bzw. funktional - nicht funktional. Keine dieser Unterscheidungen ist die „wahre“ oder ,angemessene“ Unterscheidung, sondern sie ist an die Beobachtungsperspektive gebunden und den Beobachtungsoperationen mitgegeben. Keine Beobachtung kann sich im Moment der Beobachtung selbst beobachten: Eine Beobachtung kann eine bestimmte Unterscheidung gebrauchen, aber nicht im gleichen Moment beobachten und gebraucht somit ihre Unterscheidung als ihren blinden Fleck, der erst in einer zweiten Beobachtung mit einer anderen Unterscheidung bezeichnet werden kann. Beobachtungen zweiter Ordnung beziehen sich auf eigene Unterscheidungen; die „zweite Ordnung“ gilt nur in Bezug auf eine andere Ordnung und hat an sich keine privilegierte Position (weil sie ihren eigenen blinden Fleck hat). Der „zweite“ Beobachter kann nur den blinden Fleck des „ersten“ Beobachters beobachten. In Luhmanns eigenen Worten: Der Ideologiekritiker ist nicht besser dran als der Ideologe. Er hat aber dem Beobachter erster Ordnung voraus, dass er Rückschlüsse auf 
seine eigenen Beobachtungsoperationen ziehen und seinen eigenen Standpunkt relativieren kann - er sieht zumindest, dass er nicht sehen kann, was er nicht sehen kann. In der Stafette der Beobachtungsordnungen hat die Sprachkritik ohne Sprache die Funktion, die Wahrnehmung der gesellschaftlichen Differenzen zu sichern und auf diese Weise die Stabilisierung der „richtigen Sprecher“ herzustellen. Die Beobachtung zweiter Ordnung konzentriert sich auf die gesellschaftliche und sprachliche Funktionalität. $\mathrm{Da}$ es Beobachtungsordnungen beliebiger Komplexität geben kann, könnte man auch fragen, ob es eine Beobachtung dritter Ordnung gibt. Es bietet sich eine Beobachtung von einem transzendenten Gesichtspunkt aus an, also von einem Punkt aus, der die absolute Übereinstimmung (oder NichtÜbereinstimmung) von Sprache und Gesellschaft garantiert, eine Art paradiesische Ursprache, die im Lauf der Menschheitsgeschichte verlassen wurde. Vielleicht kann man so die Sprachkritik von Karl Kraus verstehen: Kraus hätte in dieser Sicht alle Beobachtungsstufen eingenommen und als Ganzes dargestellt (und damit auch einige Verwirrung angerichtet).

5.2 Sprachkritik erster Ordnung braucht Differenzen. Differenzen kommen aus verschiedenen Substraten und Entwicklungen. Allen diesen Differenzen ist gemeinsam, dass sie aus der Störung einer ursprünglichen Stabilität entstanden sind. In grober Vereinfachung kann man davon ausgehen, dass diese ursprüngliche Stabilität literatursprachlich geprägt ist und im Deutschen in der Sprache der Weimarer Klassik am reinsten ausgeformt ist. Störungen kommen aus den bekannten Existenzformen der Sprache: diatopisch - die zunehmende Beteiligung von regionalen Sprachformen als Verkehrsdialekte mit großräumiger Geltung; diaphasisch - die zunehmende Beteilung von funktionalen Varietäten, also etwa Fachsprachen und Gruppensprachen bis hin zum Jargon; diastratisch - die zunehmende Beteilung unterschiedlicher sozialer Schichten (z.B. Jugend/Alter, Männer/Frauen usw.); diamedial - die zunehmende Beteiligung von verschiedenen medialen Kanälen (Zeitung, Rundfunk, Neue Medien, Fanzines usw.); diakonzeptuell - die zunehmende Beteiligung mündlicher Sprachformen an der grammatischen Ausformung der Schriftsprache; manche dieser Existenzformen kann man vielleicht unter bestimmten stilistischen Ebenen und Schichten zusammenfassen („Diastilistik“). Die zunehmende Komplexität der Gesellschaft (ihre polykontextuale Strukturiertheit) spiegelt sich in der zunehmenden Komplexität des Sprachverhaltens: Es ist in der heutigen Schriftsprache viel mehr üblich, akzeptiert, „erlaubt“ als zur Zeit der Weimarer Klassik. Besonders deutlich ist das am Beispiel der Wissenschaftssprache und Sonderformen wie Parlando in manchen Domänen. Dazu kommt noch eine diachronische Komponente, nicht nur in Bezug auf innersprachliche systembedingte Entwicklungen, sondern vor allem auch durch gesellschaft- 
liche Veränderungen. Besonders deutlich ist das in der neueren Sozialgeschichte ab dem Zweiten Weltkrieg. Mindestens beteiligt sind Wertewandel (traditionelle $\rightarrow$ materielle $\rightarrow$ postmaterielle Grundorientierung) und die vermehrte Beteiligung von verschiedenen gesellschaftlichen Schichten aus dem alternativen und hedonistischen Milieu (Schulze 2000, S. 335 ff.). Alle diese Veränderungen führen zu neuen sprachlichen Verhaltensweisen, die man vielleicht zusammenfassend als ,sprachliches Aufmerksamkeitsmanagement" bezeichnen kann. Sehr deutlich ist das in den Formen der Werbung und der politischen Sprache. Besonders problematisch ist das überall dort, wo eine Ausgleichssprache unerlässlich ist oder für unerlässlich gehalten wird, also in der Schule, in der öffentlichen Kommunikation, in vielen institutionellen Bereichen, und genau dort liegt das angestammte Gebiet sprachkritischer Aktionen.

5.3 Sprachkritik enthält schon in einem ihrer Wortbestandteile, der Kritik, eine Bedeutungskomponente der Unterscheidung. Insofern ist die Systemtheorie geradezu für das Verständnis der sprachkritischen Aktionen und ihrer Akteure vorausbestimmt. Die Theorie der Ordnung von Beobachtungsstufen erlaubt den Ansatz einer hierarchischen Schichtung von Unterscheidungsaktionen, so etwa wie sie Roland Barthes in seinen „Mythen des Alltags" dargestellt hat: Aus Bedeutendes und Bedeutetes entsteht ein Zeichen, das in einem sekundären semiotischen System, dem Mythischen, wieder ein Bedeutetes für ein anderes Zeichen werden kann. Systemtheoretisch kann sich diese Schichtung prinzipiell immer wieder aufbauen. Versuchsweise gebrauche ich für das Mythos-System der Sprachkritik den Terminus ,Aufklärung`. In dieser Sicht ordnen sich die verschiedenen Aufklärungsstufen ausgehend von grammatischer/lexikalischer Aufklärung (mit der Korrelation falsch - richtig, also die herkömmlichen Sprachratgeber) über linguistische Aufklärung (Korrelation sprachlich funktional - sprachlich nicht funktional, also die Vertreter der Metakritik) bis zur hier vorgestellten soziologischen Aufklärung (Korrelation gesellschaftlich funktional - gesellschaftlich nicht funktional). Da es ja um Polaritäten geht, kommt ein weiteres strukturalistisches Konzept ins Spiel: Ethik und Moral. Luhmann unterscheidet bekanntlich zwischen Ethik, die das Verhalten des Menschen im Alltagsleben lenkt, und Moral, welche als besondere Art von Kommunikation als Kode mit der Unterscheidung von gut - schlecht oder gut - böse operiert. Ethik ist in dieser Sicht eine Reflexionstheorie der Moral. Die Moral selbst liefert Hinweise auf Achtung und Missachtung in Bezug auf die Person als Ganze. Der moralische Kode ist universal und kann auf jedes Thema, jede Handlung, jede Ansicht angewendet werden. Hier lohnt sich ein Zitat (Luhmann 1990, S. 18): 
Achtung und Missachtung wird typisch nur unter besonderen Bedingungen zuerkannt. Moral ist die jeweils gebrauchsfähige Gesamtheit solcher Bedingungen. Sie wird keineswegs laufend eingesetzt, sondern hat etwas leicht Pathologisches an sich. Nur wenn es brenzlig wird, hat man Anlass, die Bedingungen anzudeuten oder gar explizit zu nennen, unter denen man andere bzw. sich selber achtet oder nicht achtet.

Auf die Ethik als Begründungstheorie der Moral will ich hier nicht eingehen. Beachtenswert ist jedenfalls, dass Luhmann moralische Kommunikation als problematisch ansieht: Sie ist oft gekennzeichnet durch einen besonderen Eifer und durch besondere Hartnäckigkeit der moralischen Bewertungen. Daher münden solche Kommunikationen auch oft in Auseinandersetzungen und Gewalt. In diesem Zusammenhang ist es aber evident, dass moralische Kommunikation zunimmt, vor allem in den Medien: Sie haben nach Luhmann einen gewaltigen Verbrauch von Moral, und zwar einer Moral, die im normalen Leben nicht gebraucht wird. Das hat Günther Pflug als „Ausweitung der Wirklichkeit“ beschrieben und den Wandel der Ethik zu einer Moral der Medien am Beispiel der Idee der Menschenrechte gezeigt. Kurz zusammengefasst sind es Moralisierung, Emotionalisierung und Verbildlichung problematischer Chrakakteristika der Mediensprache, welche die Informationsstruktur verändert haben (Pflug 2000).

5.4 Es gibt aber auch eine strukturimmanente Störstelle in der Hierarchie der Beobachtungsstufen höherer Ordnung dadurch, dass sich die Beobachtung immer auf den Kode der unteren Ordnung beziehen muss. Barthes (1964, S. 108) formuliert das sehr deutlich: „Das Störende im Mythos [ist] gerade, dass seine Form motiviert ist." Jeder Mythos gründet sich auf eine motivierende Form, Sinn wird in Form verwandelt, deformiert, seiner Geschichte beraubt:

Das Widerwärtige im Mythos ist seine Zuflucht zu einer falschen Natur, ist der Luxus der bedeutungsvollen Formen, wie bei jenen Objekten, die ihre Nützlichkeit durch einen natürlichen äußeren Schein dekorieren. Der Wille, die Bedeutung durch die ganze Bürgschaft der Natur schwerer zu machen, ruft eine Art von Ekel hervor: der Mythos ist zu reich, und gerade seine Motivierung ist zuviel an ihm.

Hier geht es zwar um die Ästhetik des Kunstwerks, dieser Gedanke lässt sich aber auf alle strukturalistischen Operationen beziehen. Wenn die Beobachtungen den Kode falsch/richtig enthalten, dann muss eine bestimmte Form gemeint sein, welche die Unterscheidung motiviert. Dieser „Überschuss" der bedeutungsvollen Formen stört das Ideal der strukturalistischen Sprachauffassung, die Lehre von der Beliebigkeit des sprachlichen Zeichens. Das einzelne Zeichen wird damit seiner idealen Funktionalität entfernt und mit symbolischem Sinngehalt verunreinigt. Das ist besonders deutlich an der Kritik von einzelnen Wörtern und Phrasen, wie z.B. vor Ort: Dann ist 
nicht mehr der Wert im einzelnen Sprachzug gemeint, sondern die „falsche" Verallgemeinerung eines fachsprachlichen Ausdrucks. Ein weiteres Beispiel ist die Kritik an Ausdrücken wie Wann geht der nächste Zug mit dem Hinweis, dass Züge nicht gehen können.

\section{Die ordentliche Sprache und ihre Gesellschaft}

6.1 Die Kräfte der deutschen Sprachkritik in einer Liste aufzählen, wäre für einen Erklärungsversuch zu wenig. Vielmehr muss ihre Stellung im Gefüge der Leistungen und Wirkungen von Sprachbeobachtungen berücksichtigt und ihr Zusammenwirken mit moralischen Einstellungen und symbolischen Prozessen gesehen werden: Einstellungen und Prozesse, die selbst wieder aus dem komplexen Gefüge der Gesellschaft entstehen und damit auch diese Gesellschaft stabilisieren. Ich zeige das an einem besonders deutlichen Fall. Durch einen befreundeten Rundfunkredakteur hatte ich die Möglichkeit, mit Erlaubnis des Österreichischen Rundfunks alle Hörerbriefe auszuwerten, die anlässlich einer Sendung in der Serie „Moment - Leben heute“ am 9. Feber 1993 mit dem Thema „Sprachpolizei“ die Rundfunkredaktion erreichten. Ich habe weiterhin einige der Autoren mit der Bitte um zusätzliche Beobachtungen angeschrieben. Auf diese Weise war es mir möglich, ein ziemlich vollständiges Bild über die Reaktionen zu dieser Sendung und darüber hinaus zur sprachlichen Einstellung mancher Leserbriefschreiber zu gewinnen. Die Auswertung (Schrodt 1997) ergab zum großen Teil bekannte Erscheinungen des Sprachwandels und der Unsicherheiten im heutigen Deutsch. Sie betreffen z.B. die Morphologie des Partizips II (eingeschalten, gewnnken, gewunschen statt eingeschaltet usw., brauchen ohne zu), Flexionsveränderungen wie eine Operation am Herz statt am Herzen, Unsicherheiten im Genus des Artikels (der shop, der service, der corpus, der virus statt das), wobei öfters unzutreffende Behauptungen vorgebracht wurden. Sehr oft stoßen Verschmelzungen wie am auf Ablehnung, wobei manchmal abenteuerliche Erklärungsversuche vorgebracht werden (die Ferien am Land wären eigentlich „neben dem Land“, richtig müsste es auf dem Land heißen). Die constructio ad sensum wie das Künstlerehepaar zeigte ihre Bilder wird abgelehnt, ebenso Dativkonstruktionen wie ich werde dem Gefübl nicht Herr. Die Flexionslosigkeit von Maßangaben wie Meter wird ebenfalls fälschlicherweise bemängelt. Ein bekanntes Gebiet der Sprachpflege ist auch der Konjunktiv. Hier wird die würde-Umschreibung in der indirekten Rede nur für den Zukunftsbezug für richtig gehalten; der Leserbriefautor sendet sogar eine Kopie des Zweifelsfälle-Dudens mit - in der Duden-Grammatik findet sich diese Vorschrift allerdings nur bis zur 3. Auflage (S. 112, \$254), ab der 4. Auflage gibt es keine entsprechenden Hinweise mehr. Auch der Dativ nach Datumsangaben (am Freitag, dem ...) wird für falsch gehalten. An falschen Kasus- 
formen werden ebenfalls moniert: während, binnen, statt, wegen + Dativ; laut, dank + Genitiv; kosten, lehren + Dativ. Das alles sind längst bekannte angebliche Sprachverfallserscheinungen. Bemerkenswert ist aber, dass die meisten Briefe von älteren Menschen geschrieben wurden. So nennt sich eine Briefschreiberin selbst eine 79-jährige „Grufty“. In einer Unterschrift heißt es Ibre alte und woblmeinende Hörerin, und einmal findet sich die Bemerkung besonders von älteren Menschen. Die Kennmelodie der Sendung wurde besonders oft abgelehnt: Dazwischen babt ibr uns ältere Hörer aber echt verulken u. ärgern wollen. Auch aus der Handschrift kann man gelegentlich auf ein höheres Alter schließen. Und manchmal werden Bemerkungen auf vergilbte Postkarten geschrieben, deren Frankierung auf die Herkunft aus den 50er-Jahren weist. Ein schöner Ausdruck von Nostalgie findet sich auch einmal in einem Brief, in dem die Schreiberin aus Meran als alte und begeisterte Zugfabrerin die neuen Hochgeschwindigkeitsstrecken ablehnt: Werden auch wir in Österreich bald mit dem Äquivalent der französischen TGV „,beglückt" werden? Entsetəlicher Gedanke! Ich persönlich fahre da lieber mit einer Postkutsche über Land. auch nach Rom. Da geht es schon längst nicht mehr um die Sprache. In vielen dieser Briefe finden wir Einstellungen, die typisch sind für die ältere Generation. Die Einstellungen dieser Generation sind besonders stark an gesellschaftlich akzeptierten Normen und Werten orientiert und haben die Funktion, eigene Orientierungen gegen die Infragestellung durch die Jungen zu verteidigen. Sie ermöglichen den Gewinn von Selbstbehauptung und Selbstachtung (Ich-Verteidigungs-Funktion). Solche Äußerungen sind damit vielleicht weniger ein Mittel der Kritik, sondern vielmehr der pointierten Selbstdarstellung und darüber hinaus ein Element der pseudorationalen Bewältigung der Lebenssituation dieser Generation (Kaiser 1991, S. 179); sie finden direkt Anschluss an die soziologischen Befunde zum Lebenszyklus und zur Gegenkultur (Schulze 2000, S. 366 f., 370 f.).

6.2 Die Kräfte der deutschen Sprachkritik zeigen sich auch an diesem Beispiel. Es sind mindestens beteiligt: Alter, Bildung, gesellschaftlicher Stand, öffentlicher Raum, Faktoren des Aufmerksamkeitsmanagements seitens des Kritisierten, Faktoren des Kulturkonservatismus seitens des Kritisierenden. $\mathrm{Da}$ ist die Sprache gar nicht mehr so wichtig. Sprachkritik ist das Feld, in dem komplex geschichtete Hierarchien von Beobachtungen die gesellschaftlich wirksamen Differenzen bezeichnen - Bezeichnungen, welche die Stabilität der Gesellschaft immer wieder herstellen. Insofern ist Sprachkritik durchaus nützlich - wenn man bedenkt, dass es hier gar nicht um die Sprache geht. Dass am Anfang dieser Beobachtungsschichten einmal die Sprache beobachtet wurde, wird dann irgendwann einmal Geschichte.

Dass Sprachkritik mit historischen Ereignissen und gesellschaftlichen Umbrüchen verbunden ist, hat von Polenz (1999, S. 294 ff.) eindrucksvoll zusammenfassend gezeigt. Sprachkritik ist aber auch mit einem synchronen 
Wirkungsgefüge gesellschaftlicher Schichten verknüpft, für das sich Ludwig Wittgensteins Begriff der „Lebensform“ besonders gut eignet. Das Bemühen um soziale Distinktion durch die Sprachbeobachtung kann in einem innerbürgerlichen Konkurrenzkampf als treibende Kraft für das Herausbilden verschiedener, komplex geschichteter Beobachtungsordnungen verstanden werden - Ordnungen, welche die Gesellschaft gerade in ihren prekären, hybriden, durchlässigen und dynamischen Schichtungen zu einem Mindest$\mathrm{maß}$ an Stabilität verhilft. Sprachkritik verhilft dazu, die komplexen Bruchlinien unserer Gesellschaft deutlich zu machen, und eben diese Deutlichkeit ist für selbstbestimmtes Orientieren und Handeln ein entscheidender Vorteil, manchmal wohl auch eine Notwendigkeit. Systemtheoretisch gesehen sind die Formen von Sprachkritik ein heuristisch wertvolles Instrument zur Untersuchung und Erklärung der „Kräfte des menschlichen Daseins“, wie es Weisgerber seinerzeit formuliert hat - wenn es gelingt, Mythos und Wirklichkeit zu unterscheiden.

\section{Quellen}

Marcus Fabius Quintilianus (1972): Ausbildung des Redners. 12 Bücher. Hrsg. u. übers. v. Helmut Rahn. Erster Teil. Darmstadt.

te Neues Verlag (o.J.) (Hg.): MAX City Guide Hamburg.

\section{Literatur}

Ágel, Vilmos (2008): Bastian Sick und die Grammatik. Ein ungleiches Duell. In: Informationen DaF 35, S. 64-84.

Androutsopoulos, Jannis (2005): ... und jetzt gehe ich chillen: Jugend- und Szenesprachen als lexikalische Erneuerungsquellen des Standards. In: Eichinger, Ludwig M./Kallmeyer, Werner (Hg.): Standardvariation. Wie viel Variation verträgt die deutsche Sprache? (= Jahrbuch des Instituts für Deutsche Sprache 2004). Berlin.

Barthes, Roland (1964): Mythen des Alltags. Frankfurt a.M.

Canoğlu, Hatice Deniz (2012): Kanak Sprak versus Kiezdeutsch - Sprachverfall oder sprachlicher Spezialfall? Eine ethnolinguistische Untersuchung. (= Sprachwissenschaft 11). Berlin.

Duden (2002): Der Duden. Bd. 9: Richtiges und gutes Deutsch. Mannheim.

Duden (2009): Der Duden. Bd. 4: Die Grammatik. Unentbehrlich für richtiges Deutsch. 8., überarb. Aufl. Mannheim/Wien/Zürich.

Dürscheid, Christa/Neuland, Eva (2006): Spricht die Jugend eine andere Sprache? Neue Antworten auf alte Fragen. In: Dürscheid/Spitzmüller (Hg.), S. 19-32.

Dürscheid, Christa/Spitzmüller, Jürgen (Hg.) (2006): Perspektiven der Jugendsprachforschung. (= Sprache, Kommunikation, Kultur 3). Frankfurt a.M. u.a. 
Elspaß, Stephan (2005): Sprachgeschichte von unten. (= Reihe germanistische Linguistik 263). Tübingen.

Engel, Ulrich (1988): Deutsche Grammatik. Heidelberg.

Engel, Ulrich (2004): Deutsche Grammatik. Neubearbeitung. München.

Gaar, Emil/Schuster, Mauritz (o.J.): Lateinische Grammatik. 13. Aufl. Wien.

Gelhaus, Hermann (1972): Synchronie und Diachronie. Zwei Vorträge über Probleme der nebensatzeinleitenden Kunjunktionen und der Consecutio temporum. (= Europäische Hochschulschriften 1; 72). Bern/Frankfurt a.M.

Gelhaus, Hermann (1974): Untersuchungen zur Consecutio temporum im Deutschen. In: Gelhaus, Hermann/Latzel, Sigbert: Studien zum Tempusgebrauch im Deutschen. (= Forschungsberichte des Instituts für deutsche Sprache 15). Mannheim, S. 1-127.

Helbig, Gerhard (1970): Geschichte der neueren Sprachwissenschaft. Leipzig.

Jung, Walter (1980): Grammatik der deutschen Sprache. 6., neubearb. Aufl., Neuausg. Leipzig.

Kaiser, Heinz Jürgen (1991): Generationsprobleme. In: Oswald, Wolf D. et al. (Hg.): Gerontologie. Medizinische, psychologische und sozialwissenschaftliche Grundbegriffe. 2., überarb. u. erw. Aufl. Stuttgart/Berlin/Köln, S. 177-185.

Klein, Wolfgang (1986): Der Wahn vom Sprachverfall und andere Mythen. In: Zeitschrift für Literaturwissenschaft und Linguistik 62, S. 11-28.

Kneer, Georg/Nassehi, Armin (1997): Niklas Luhmanns Theorie sozialer Systeme. Eine Einführung. 3., unveränd. Aufl. (= UTB: Soziologie, Philosophie, Literaturwissenschaft 1751). München.

Kowal, Jerzy (2007): Spanish consecutio temporum: myths and reality. (= LINCOM Studies in Romance Linguistics 52). München.

Kühner, Raphael/Stegmann, Carl (1976): Ausführliche Grammatik der lateinischen Sprache. Zweiter Teil: Satzlehre, Bd. 2. 5. Aufl. Darmstadt.

Luhmann, Niklas (1990): Paradigm lost. Über die ethische Reflexion von Moral. 2. Aufl. Frankfurt a.M.

Luhmann, Niklas (2012): Soziale Systeme. Grundriß einer allgemeinen Theorie. 15. Aufl. Frankfurt a.M.

Mackowiak, Klaus (2008): Die 101 häufigsten Fehler im Deutschen und wie man sie vermeidet. 3., aktual., neu bearb. und erw. Aufl. München.

Maitz, Péter (2010): Sprachpflege als Mythenwerkstatt und Diskriminierungspraktik. In: Aptum 6, S. 1-19.

Mattheier, Klaus J. (1995): Sprachgeschichte des Deutschen: Desiderate und Perspektiven. In: Gardt, Andreas/Mattheier, Klaus J./Reichmann, Oskar (Hg.): Sprachgeschichte des Neuhochdeutschen. Gegenstände, Methoden, Perspektiven. (= Reihe Germanistische Linguistik 156). Tübingen, S. 1-18. 
Matthias, Theodor (1929): Sprachleben und Sprachschäden. Ein Führer durch die Schwankungen und Schwierigkeiten des deutschen Sprachgebrauchs. 6., verb. u. verm. Aufl. Leipzig.

Meinunger, André (2008): Sick of Sick? Ein Streifzug durch die Sprache als Antwort auf den „Zwiebelfisch“. 2. Aufl. Berlin.

Minor, Jakob (1892): Allerhand Sprachgrobheiten. Eine höfliche Entgegnung. Stuttgart.

Neuland, Eva (2008): Jugendsprache. Eine Einführung. (= UTB 2397). Tübingen.

Neuland, Eva (2011): Jugendliche als Sprachakteure: Formen sprachlicher Stilbildung und sozialer Distinktion. In: Baurmann, Jürgen/Neuland, Eva (Hg.): Jugendliche als Akteure. Sprachliche und kulturelle Aneignungs- und Ausdrucksformen von Kindern und Jugendlichen. (= Sprache, Kommunikation, Kultur 9). Frankfurt a.M. u.a., S. 13-24.

Neuland, Eva (Hg.) (2012): Sprache der Generationen. (= Thema Deutsch 12). Mannheim.

Pflug, Günther (2000): Mediensprache zwischen Neugier und Moral. In: EichhoffCyrus, Karin M./Hoberg, Rudolf (Hg.): Die deutsche Sprache zur Jahrtausendwende. Sprachkultur oder Sprachverfall? (= Thema Deutsch 1). Mannheim u.a.

Polenz, Peter von (1999): Deutsche Sprachgeschichte vom Spätmittelalter bis zur Gegenwart. Bd. 3: 19. und 20. Jahrhundert. Berlin u.a.

Rheindorf, Markus (2011): Wandel der deutschen Sprache: Eine textsortenbezogene Pilotstudie. Internet: www.univie.ac.at/linguistics/publikationen/WandelderSprachePilot studie.pdf (zitiert als Rheindorf 1); images.derstandard.at/2012/04/25/Pilotstudie_ Sprachwandel.pdf (zitiert als Rheindorf 2) (Stand: 11.4.2013).

Romberg, Johanna (2012): Was reden wir denn da? In: GEO 11/2012, S. 136-149.

Sanders, Daniel (1908): Wörterbuch der Hauptschwierigkeiten in der deutschen Sprache. 39.-40. Aufl. Berlin.

Schlobinski, Peter (Hg.) (2006): Von *hdl* bis *cul8r*. Sprache und Kommunikation in den Neuen Medien. (= Thema Deutsch 7). Mannheim u.a.

Scholes, Robert (1974): Structuralism in literature. An introduction. New Haven/ London.

Schrodt, Richard (1995): Warum geht die deutsche Sprache immer wieder unter? Die Problematik der Werthaltungen im Deutschen. (= Passagen Diskursforschung). Wien.

Schrodt, Richard (1997): „Sprache ist etwas Lebendiges, doch falsche Ausdrücke haben damit nichts zu tun“ - oder: „Wenn man nur wüßte, wann die „Sprachpolizei“ wieder kommt!“ Bemerkungen zu sprachkritischen Leser- und Hörerbriefen. In: Brandt, Gisela (Hg.): Historische Soziolinguistik des Deutschen. Bd. 3: Sprachgebrauch und sprachliche Leistung in sozialen Schichten und soziofunktionalen Gruppen. Internationale Fachtagung, Rostock, Kühlungsborn 15.-18.9.1996. (= Stuttgarter Arbeiten zur Germanistik 351). Stuttgart, S. 299-314. 
Schrodt, Richard (1998): Zeitenfolgeregeln sind Aspektkongruenzregeln: Bemerkungen zur Diachronie des deutschen Perfekts. In: ZAS Papers in Linguistics 13, S. 240-242.

Schrodt, Richard (2007): Temporalität beim Konjunktiv? In: Lenk, Hartmut E.H./ Walter, Maik (Hg.): Wahlverwandtschaften: Valenzen - Verben - Varietäten. Festschrift für Klaus Welke zum 70. Geburtstag. (= Germanistische Linguistik 188/ 189). Hildesheim/Zürich/New York, S. 247-260.

Schulze, Gerhard (2000): Die Erlebnis-Gesellschaft. Kultursoziologie der Gegenwart. 8. Aufl. Frankfurt a.M.

Sick, Bastian (2005): Der Dativ ist dem Genitiv sein Tod. Folge 2: Neues aus dem Irrgarten der deutschen Sprache. Köln.

Sick, Bastian (2006a): Der Dativ ist dem Genitiv sein Tod. 25. Aufl. Köln/Hamburg.

Sick, Bastian (2006b): Der Dativ ist dem Genitiv sein Tod. Folge 3: Noch mehr Neues aus dem Irrgarten der deutschen Sprache. Köln.

Spitzmüller, Jürgen (2006): Der mediale Diskurs zu ,Jugendsprache‘: Kontinuität und Wandel. In: Dürscheid/Spitzmüller (Hg.), S. 33-50.

Trabold, Annette (1993): Sprachpolitik, Sprachkritik und Öffentlichkeit. Anforderungen an die Sprachfähigkeit des Bürgers. Wiesbaden.

Vernaleken, Theodor (1900): Deutsche Sprachrichtigkeiten und Spracherkenntnisse. Wien.

Volmert, Johannes (2006): Jugendsprachliche Stile und Register. Einfluss auf und Stellenwert für mündliche und schriftliche Kommunikation im Unterricht. In: Dürscheid/Spitzmüller (Hg.), S. 87-100.

Wahrig (2009): Richtiges Deutsch leicht gemacht. (= Wahrig 5). Gütersloh/München.

Weisgerber, (Johann) Leo (1954): Die Sprache unter den Kräften des menschlichen Daseins. 2. Aufl. (= Von den Kräften der deutschen Sprache 1). Düsseldorf.

Wierzbicka, Mariola/Schlegel, Dorothee (2008): Sprechzeiten im Diskurs. Zum absoluten und relativen Gebrauch der Tempora in der gesprochenen deutschen Sprache. München.

Wiese, Heike (2012): Kiezdeutsch. Ein neuer Dialekt entsteht. München.

Wollmann, Franz (1967): Deutsche Sprachkunde auf sprachgeschichtlicher Grundlage. 8. Aufl. Wien/Graz.

Wunderlich, Dieter (1970): Tempus und Zeitreferenz im Deutschen. (= Linguistische Reihe 5). München.

Wustmann, Gustav (1891): Allerhand Sprachdummheiten. Kleine deutsche Grammatik des Zweifelhaften, des Falschen und des Häßlichen. Ein Hilfsbuch für alle, die sich öffentlich der deutschen Sprache bedienen. Leipzig. 